\title{
Vaccinations for the HIV-Infected Adult: A Review of the Current Recommendations, Part II
}

Nancy F. Crum-Cianflone · Eva Sullivan

Received: June 10, 2017 / Published online: August 5, 2017

(C) The Author(s) 2017. This article is an open access publication

\begin{abstract}
Vaccination is a critical component for ensuring the ongoing health HIV-infected adults. Since this group may have reduced immune responses and shorter durations of protection post-vaccination, HIV-specific guidelines have been published. This review article provides a comprehensive discussion of the current guidelines and evidence-based data for vaccinating HIV-infected adults, including data on dosing schedules, immunogenicity studies, and safety. In the current paper, part II of the review, live vaccines, as well as vaccines for travelers and specific occupational groups, will be discussed.
\end{abstract}

Enhanced content To view enhanced content for this article go to http://www.medengine.com/Redeem/ 61E8F06021EA7287.

N. F. Crum-Cianflone $(\bowtie)$

Internal Medicine Department, Scripps Mercy

Hospital, San Diego, CA, USA

e-mail: nancy32red@yahoo.com;

Crum-cianflone.nancy@scrippshealth.org

N. F. Crum-Cianflone

Infectious Disease Division, Scripps Mercy Hospital, San Diego, CA, USA

N. F. Crum-Cianflone

Infectious Disease Division, Naval Medical Center

San Diego, San Diego, CA, USA

E. Sullivan

Pharmacy Department, Scripps Mercy Hospital, San

Diego, CA, USA
Keywords: HIV; Human immunodeficiency virus; Review; Vaccinations; Vaccine recommendations

\section{OVERVIEW}

HIV-infected persons are at an increased risk for a wide variety of infections, and hence vaccination remains an important strategy for protection of this vulnerable population. This review, part II of a two-part series (doi:10.1007/s40121-017-0166$\mathrm{x}$ ), will highlight the importance of vaccination as a critical preventive strategy among HIV-infected adults, and provide a summary of the current evidence-based recommendations including those published by advisory groups including the World Health Organization (WHO) [1, 2], British HIV Association (BHIVA) [3], and US-based groups (US Centers for Disease Control and Prevention (CDC), Advisory Committee on Immunization Practices (ACIP), and the HIV Medicine Association of the Infectious Diseases Society of America (HIVMA) [4-7]). Additionally, the European AIDS Clinical Society (EACS) guidelines [8] and French guidelines [9] will be discussed. Although published guidelines are similar in many of their recommendations, there are notable differences which will be outlined in the current paper.

Vaccines categorized as inactivated vaccines with broad indications [influenza, 
pneumococcal, HBV, and tetanus-diphtheria (Td)/tetanus-diphtheria-pertussis (Tdap)] and those advised for HIV-infected persons with additional risk factors or specific age groups [hepatitis A virus (HAV), human papillomavirus (HPV), and meningococcal] were discussed in Part I of this review. The current paper, Part II of the series, will discuss live vaccines [varicella, zoster, and measles-mumps-rubella (MMR)] (Table 1). In addition, travel-related vaccines (polio, typhoid, yellow fever, rabies, Japanese encephalitis virus, cholera, and tick-borne encephalitis) (Table 2) and vaccines to protect against specific occupational or exposure risks (anthrax, smallpox) will be reviewed (Table 3). Finally, other vaccines [Haemophilus influenzae serotype $b$ and Bacille Calmette-Guerin (BCG)] will also be reviewed (Table 3). For each vaccine, the causative infectious agent for the disease will be briefly discussed followed by the current guideline recommendations by various advisory groups (WHO, BHIVA, EACS, French, and US-based groups such as the CDC, the ACIP, and HIVMA). Data regarding post-vaccination immune responses and durability in the HIV-infected adult, as well as safety data, will then be discussed in each section. This article represents a review article and is based on previously conducted studies and does not involve any new studies of human or animal subjects performed by either of the authors.

\section{LIVE VACCINES}

Live vaccinations are generally avoided among HIV-infected persons if an inactivated form of the vaccine is available (e.g., influenza, polio, and typhoid). The reason for avoiding live vaccines among immunosuppressed persons is the concern for possible viral or bacterial replication post-vaccination and subsequent infection by the vaccine strain $[10,11]$. When only live vaccines are available for prevention of an infectious agent, some live vaccines (e.g., varicella, zoster, MMR) may be considered if the level of immunosuppression is low (CD4 count
$>200$ cells $/ \mathrm{mm}^{3}$ ) [6]. A concise summary of the guidelines regarding live vaccines among HIV-infected persons is presented in Table 1.

\section{VZV: Varicella and Zoster}

Varicella zoster virus (VZV) infections are more common among HIV-infected persons [10, 12]. This includes an elevated risk for more severe forms of chickenpox including disseminated disease. Additionally, epidemiologic data suggest that HIV-infected persons have a higher risk for VZV reactivation (i.e., shingles) with a 10 - to 20-fold higher risk noted during the pre-ART era and a continued 3- to 5-fold higher risk thereafter [3, 13-15]. Risk for VZV reactivation is related to low CD4 counts and detectable HIV viral loads [14], and manifestations may include diffuse vesicular skin lesions, ocular disease (e.g., acute retinal necrosis), encephalitis, and/or visceral dissemination.

Although varicella and zoster vaccinations are not licensed specifically for HIV-infected persons, prevention via vaccination can be considered especially given HIV-infected persons' higher risk for complicated disease. Specifically, vaccination can be considered among non-immune, clinically stable patients with a CD4 count $>200$ cells $/ \mathrm{mm}^{3}$ (CD4\% >15\%). For persons with more severe immunosuppression, vaccination is not recommended due to the risk for disseminated disease due to the live vaccine strain $[10,11]$.

Prior to deciding on varicella vaccination for HIV-infected adults, most guidelines recommend determination of the VZV immune status. Lack of VZV immunity is defined as no prior varicella vaccination, no serologic evidence of immunity (e.g., VZV IgG), and no prior clinician/laboratory diagnosed varicella or zoster infection [4]. Many HIV-infected adults have evidence of prior infection or immunity, with one study showing that 95\% of US HIV-infected adults are VZV seropositive [16] with a percentage of $>98 \%$ in the UK [17]; however, geographic variation exist [2]. Hence, a history regarding prior vaccination/infection should be obtained and, if negative, then, if possible, a 


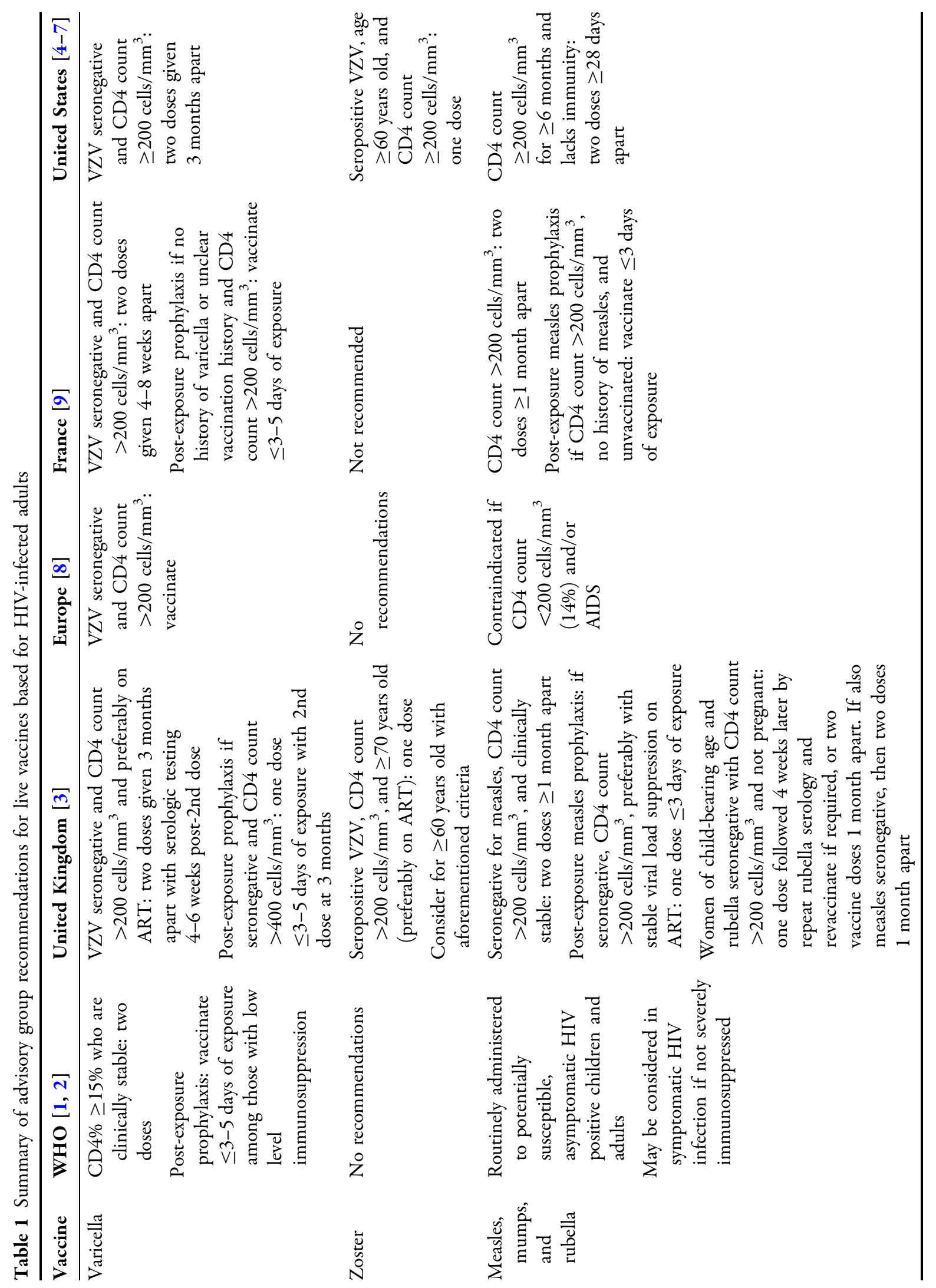




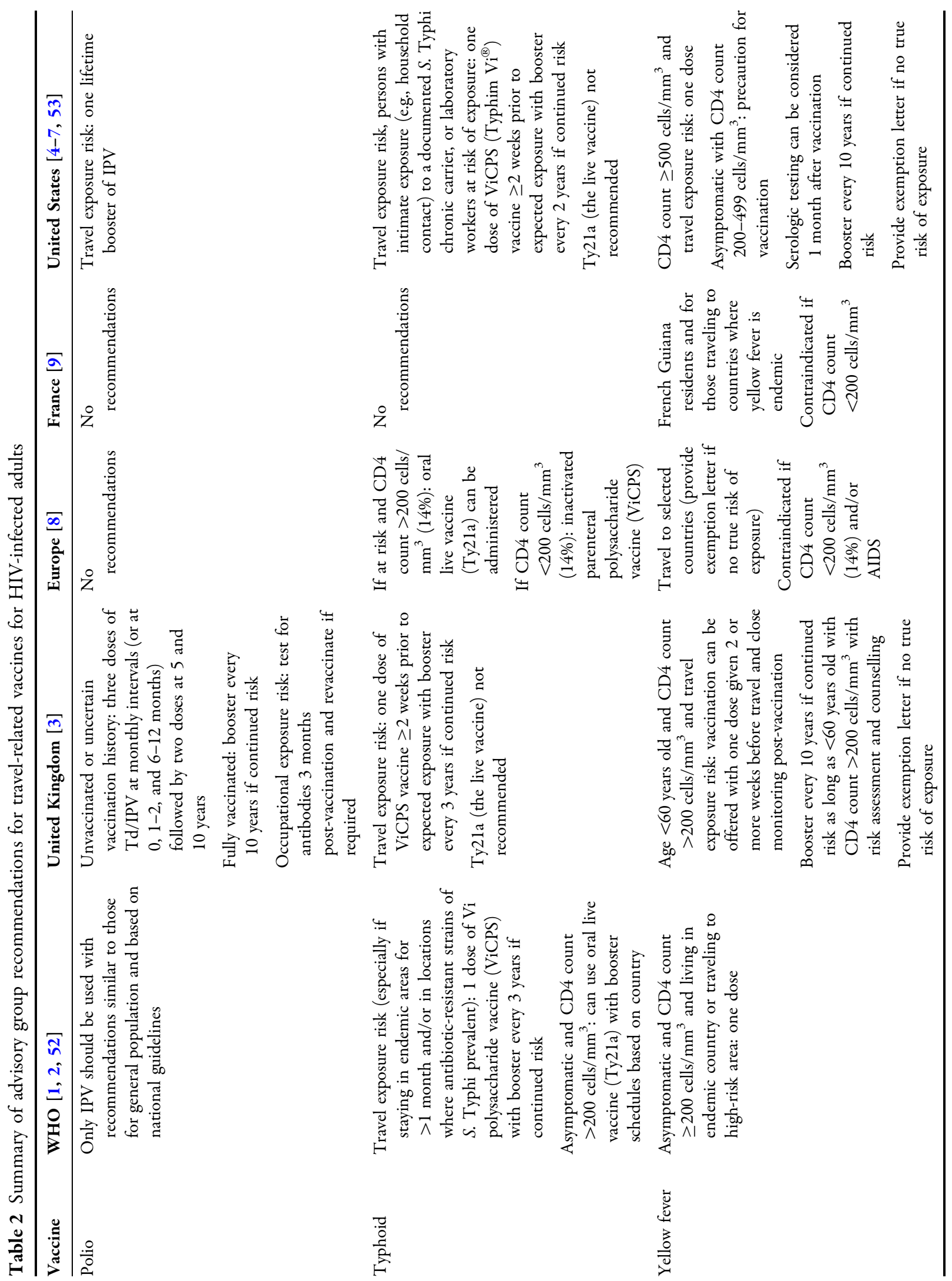




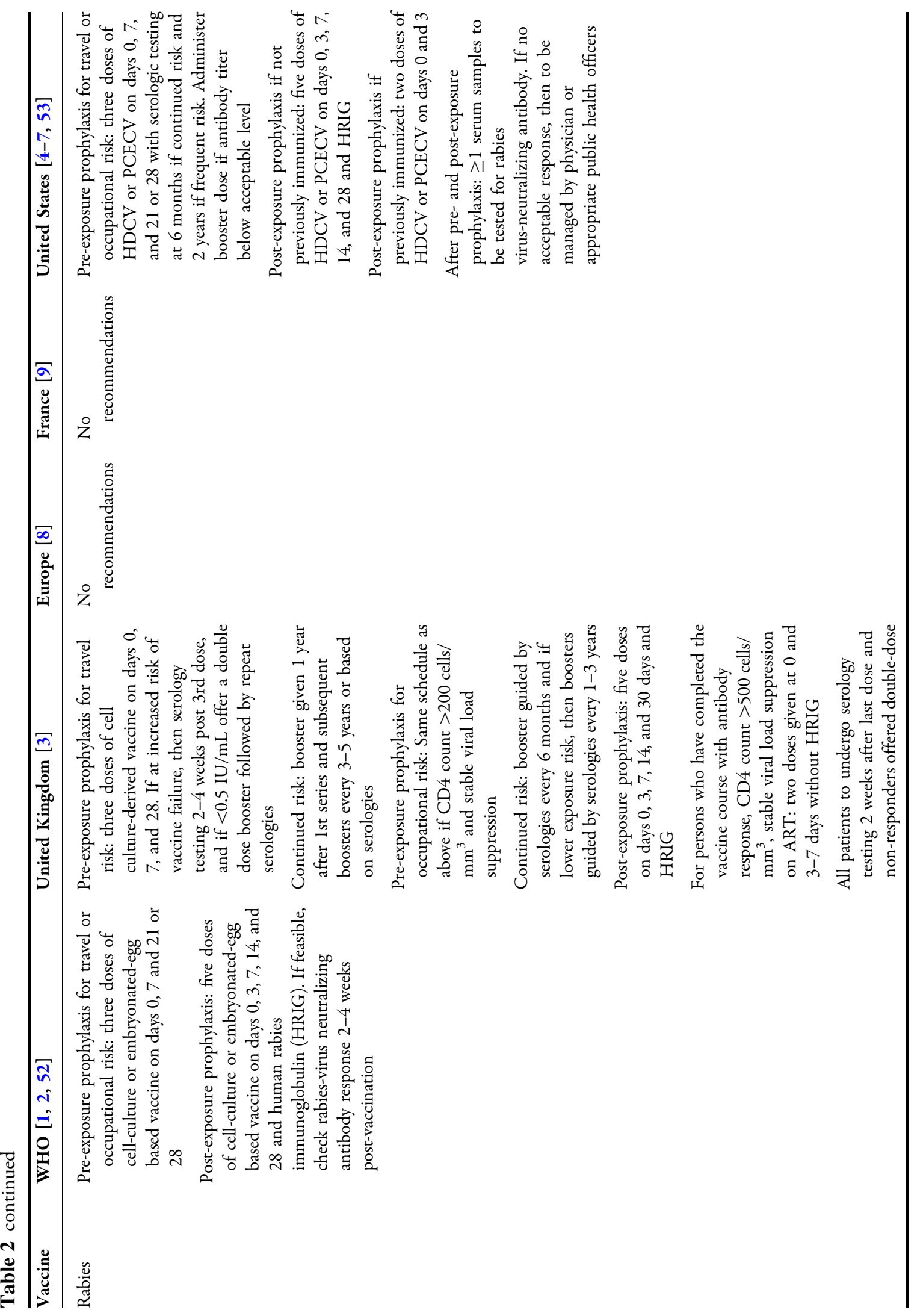




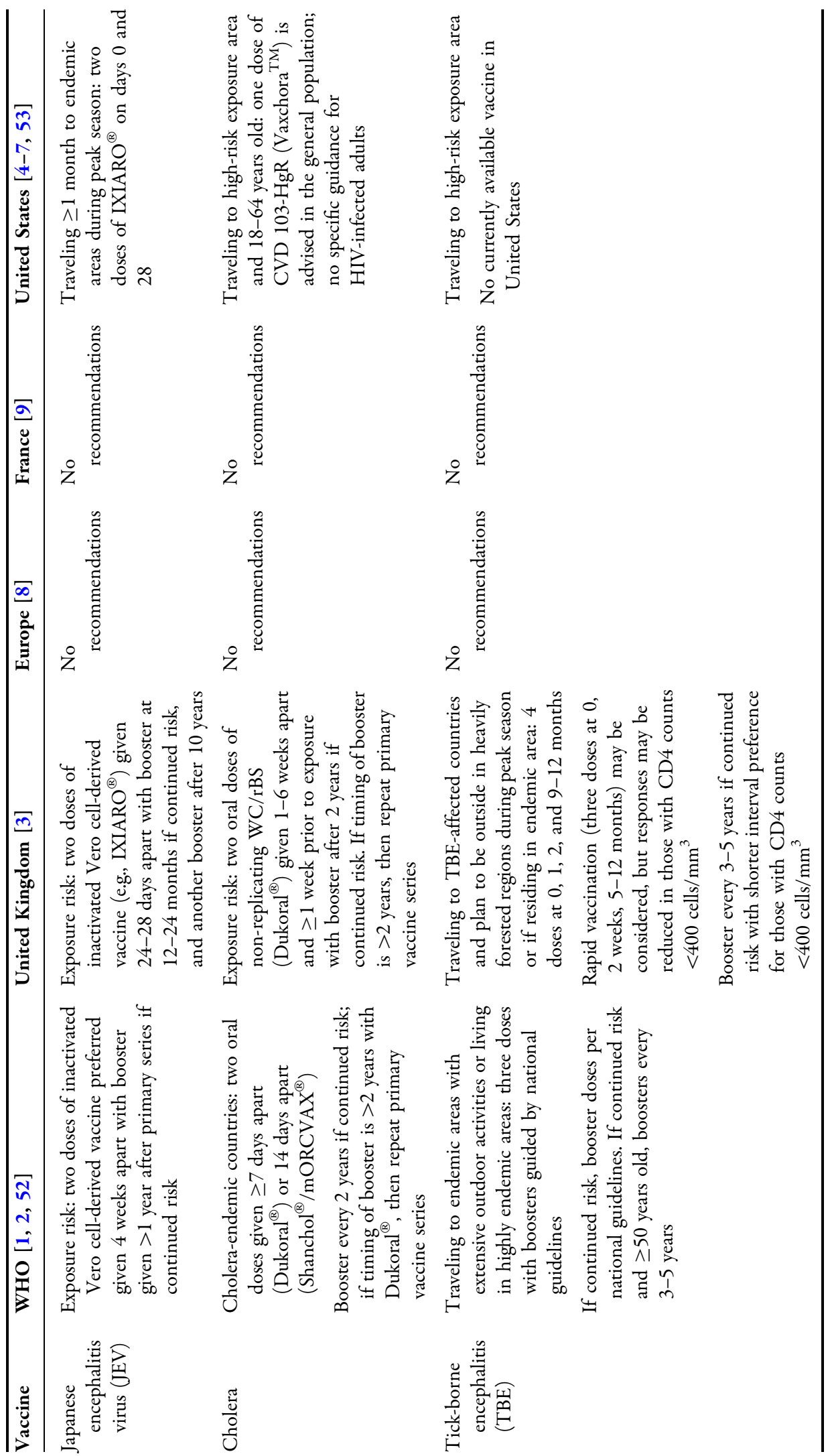




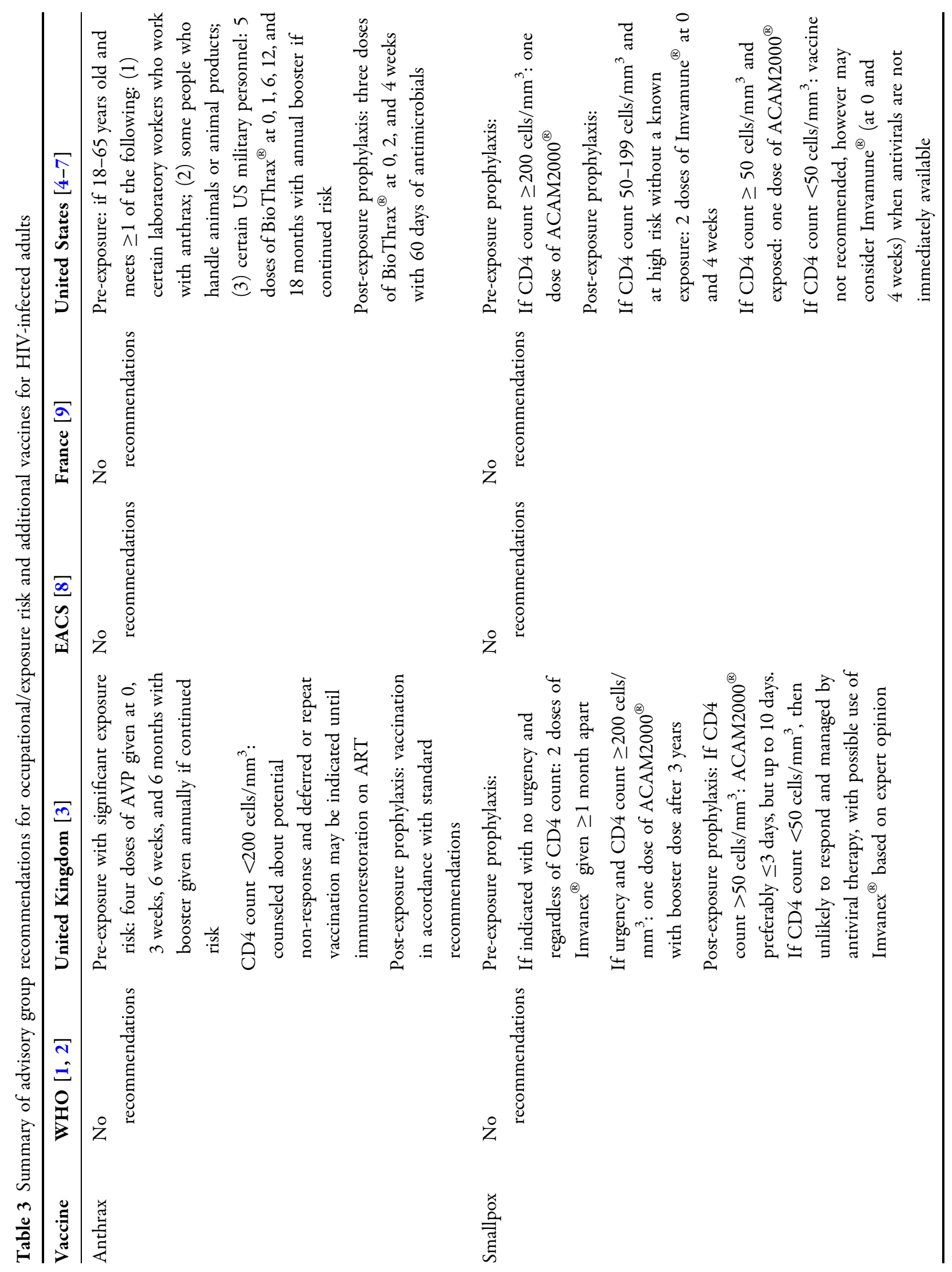




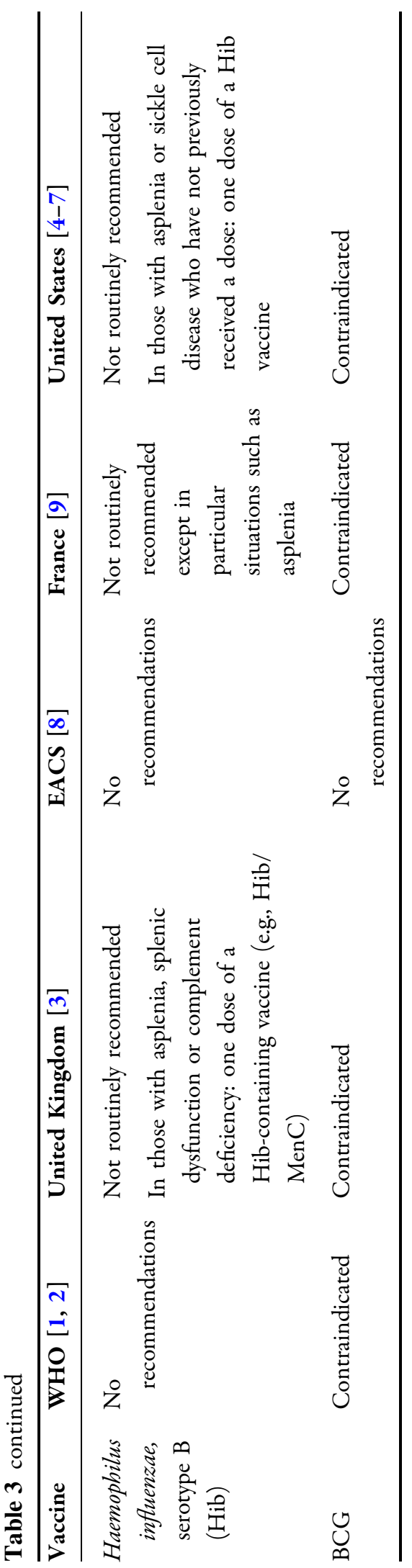

varicella IgG antibody level checked to ensure that the patient is seronegative and requires varicella vaccination. If the patient is VZV seropositive and/or has a history of prior chickenpox infection, then the zoster vaccine can be considered based on age.

Although herpes zoster disease is a serious disease with a large burden in the HIV population, a survey performed among HIV care providers in the US reported poor knowledge of the incidence of disease recurrence in HIV-infected persons and the effectiveness of herpes zoster vaccine for protection against reactivation of VZV [18]. This highlights the importance of education among HIV providers to optimize VZV prevention in this patient population.

\section{Varicella Vaccination}

WHO guidelines state varicella vaccination (given as two doses) may be administered to clinically stable HIV-infected children or adults with CD4 cell percentage $\geq 15 \%$, including those receiving ART. They recommend against vaccination for those with a CD4\% $<15 \%$ or those who are not clinically and immunologically stable. Additionally, vaccination as post-exposure prophylaxis to non-immune, exposed HIV-infected persons with low level immunosuppression can be considered and given immediately (or within 3-5 days of exposure) [1, 2]. Even though there are no available data among HIV-infected persons, data supports the use of vaccination as post-exposure prophylaxis in healthy persons [19]. The measles-mumps-rubella-varicella (MMRV) vaccine is not recommended in HIV-infected persons.

BHIVA recommends HIV-infected persons with a negative or uncertain history of chickenpox or shingles undergo VZV IgG testing. Those who are VZV seronegative with a CD4 count $>200$ cells $/ \mathrm{mm}^{3}$, and preferable on ART, can be vaccinated with two doses given 3 months apart [3]. Post-vaccination testing 4-6 weeks after the 2 nd dose is recommended for documenting VZV IgG seroconversion. Guidelines also recommend that anti-herpetic medications (e.g., acyclovir) be discontinued at the time of vaccination and for the next 4 weeks, since these medications can inhibit 
post-vaccination immune responses. Additionally, BHIVA recommends varicella vaccination to be given immediately (or within 3-5 days of exposure) as post-exposure prophylaxis to non-immune, exposed HIV-infected adults with a CD 4 count $>400$ cells $/ \mathrm{mm}^{3}$; for HIV-infected adults with lower CD4 counts, the guideline discusses the use of varicella zoster immune globulin (VZIG) and/or antiviral prophylaxis [3]. Similar to BHIVA, EACS and French guidelines also recommend varicella vaccination among non-immune persons with a CD4 count $>200$ cells $/ \mathrm{mm}^{3} \quad(>14 \%) \quad[8,9]$. Vaccination should be avoided among pregnant women.

US guidelines mirror the guidance above and state that non-immune HIV-infected adults with a CD4 count $>200$ cells $/ \mathrm{mm}^{3}$ can receive varicella vaccination (administered as a 2 -dose series with doses separated by $>3$ months) $[4,5,7]$. Guidelines recommend that anti-herpetic medications be discontinued during the period of -3 to +14 days of vaccination [5]. No recommendation for post-vaccination VZV IgG testing are included. Additionally, there are no US recommendations on post-exposure varicella vaccination in adults, as the guidelines state that, even though it has been effective in reducing the risk for varicella in VZV-susceptible immunocompetent children, it has not been established in the adolescent and adult population. Those who are susceptible to VZV and who have not been vaccinated, have no history of varicella or herpes zoster, or are seronegative for VZV, should receive post-exposure prophylaxis with VZIG as soon as possible (but within 10 days) after exposure [7].

There are currently no clinical data on the immunogenicity of varicella vaccination among seronegative HIV-infected adolescents or adults. However, studies in HIV-infected children have shown good immunogenicity, with similar antibodies levels compared to post-natural infection [20-23]. Regarding clinical efficacy among HIV-infected children without severe immunosuppression, the varicella vaccine was $82 \%$ effective in preventing varicella and $100 \%$ effective in preventing zoster in children [20]. Importantly, these data highlight that varicella vaccination also protects against future herpes zoster infections. A single study of varicella vaccinations among HIV-infected adults (CD4 count $\geq 400$ cells $/ \mathrm{mm}^{3}$ and suppressed HIV viral loads) has been conducted albeit in a cohort already VZV seropositive; the study showed that vaccination modestly boosted cellular responses [24].

Safety data in HIV-infected children have shown that local reactions occurred in 15\% after the first dose and in 10\% after the second dose, while systemic reactions including rash and headache were reported in 5\% only after the first dose [23]. In a study with VZV-seropositive HIV-infected adults with CD4 counts $\geq 400$ cells $/ \mathrm{mm}^{3}$, vaccine adverse events were mild, and were equally common among vaccine and placebo recipients [24].

\section{Zoster Vaccination}

The zoster vaccine is at least 14 times as potent as the varicella vaccine and works by boosting natural immunity to prevent VZV reactivation and subsequent shingles.

WHO did not recommend the routine use of the zoster vaccine in their 2014 guidelines, stating insufficient data regarding the burden of disease in many countries and questions regarding vaccine durability [2]. Nonetheless, countries may decide to introduce routine zoster vaccination if they have a notable burden of disease and consider vaccination beneficial. No specific guidance among HIV-infected adults is provided.

BHIVA recommends that HIV-infected adults without a reliable history of chickenpox or shingles should undergo serologic testing prior to vaccination. HIV-infected adults who are VZV IgG seropositive with a CD4 count $>200$ cells $/ \mathrm{mm}^{3}$, and preferably on ART, should be offered a single dose of the zoster vaccine in line with national indications which recommend vaccination at $>70$ years. BHIVA guidelines also recommend for the consideration of vaccination among HIV patients meeting the aforementioned criteria who are $>60$ years of age [3]. EACS guidelines do not mention zoster vaccination [8], while French guidelines state that, due to the lack of current data, vaccination is not recommended among HIV-infected persons [9]. 
In the US guidelines, a single dose of zoster vaccine can be considered among HIV-infected adults with known varicella immunity (i.e., a history of varicella or zoster, or VZV IgG positivity) who are $>60$ years of age and have clinically stable HIV infection with a CD4 count of $>200$ cells $/ \mathrm{mm}^{3}[4,7]$. Among patients with no history of varicella or zoster and having a negative VZV IgG level, providers should consider varicella vaccination as reviewed above. The need for booster doses after initial zoster vaccination is currently under study, and current guidelines have not provided any recommendations. Among the general population, waning of immunity has been noted by $\sim 8$ years post-vaccination [25], but there are no current studies regarding durability among HIV-infected adults.

Those with low CD4 counts $\left(<200\right.$ cells $/ \mathrm{mm}^{3}$ or $15 \%$ ) have the greatest risk for shingles; however, they are not recommended for vaccination given their possible risk for infection due to the live vaccine strain. Guidelines (BHIVA and US) recommend vaccinating non-immune, close contacts to protect HIV-infected persons from exposure to VZV. While there are no formal guidelines on additional strategies for preventing shingles among HIV-infected persons with high level immunosuppression (CD4 count $<200$ cells $/ \mathrm{mm}^{3}$ ), a study did find that acyclovir prophylaxis (400 mg twice daily) reduced the occurrence of shingles by $62 \%$ among HIV-infected, ART-naïve persons with CD4 counts $>250$ cells $/ \mathrm{mm}^{3}$ [26]. Subunit vaccines containing varicella-zoster virus glycoprotein $\mathrm{E}$ and the $\mathrm{ASO}_{\mathrm{B}}$ adjuvant system $[27,28]$ are in development and, since they are non-live vaccines, may have an important future role in protecting HIV-infected adults with high-level immunosuppression [29]. An inactivated zoster vaccine has also been studied in subpopulations of immunocompromised adults, including those with HIV with CD4 counts $\leq 200$ cells/ $\mathrm{mm}^{3}$, and was found to be immunogenic; however, it is still investigational and not yet available [30].

Regarding clinical efficacy, the zoster vaccine has been shown to reduce the incidence of shingles, the severity of disease, and the occurrence of post-herpetic neuralgia in the general population [31]. No clinical efficacy data exist among HIV-infected adults. Data on zoster vaccine immunogenicity include a study of 295 HIV-infected adults who were VZV seropositive which found that two doses of Zostavax ${ }^{\circledR}$ (administered 6 weeks apart) were immunogenic among HIV patients who had CD4 counts of $>200$ cells $/ \mathrm{mm}^{3}$ and undetectable HIV viral loads; those with higher CD4 counts had more robust antibody titers [32]. More data on the ideal dosing schedule, efficacy, and safety of the zoster vaccine among HIV-infected persons are needed.

No safety issues or reported cases of VZV infection by the vaccine strain have been reported to date when the vaccine was used in the recommended groups of HIV-infected persons [32, 33].

\section{Measles-Mumps-Rubella}

Cases of measles, mumps, and rubella continue to occur in both the developing and developed world, highlighting the importance of vaccination $[34,35]$. Measles infections can be particularly severe, with life-threatening infections described among HIV-infected persons [36]; clinical cases of mumps and rubella are usually similar to non-immunosuppressed hosts. Vaccination is typically with a combined vaccine containing measles-mumps-rubella (MMR).

MMR vaccination is not recommended among severely immunosuppressed HIV-infected persons (defined as CD4 count $<200$ cells $/ \mathrm{mm}^{3}$ ) due to the possibility of developing an active measles infection (e.g., pneumonia) following vaccination, as well as poorer post-vaccination immune responses $[1,37,38]$. However, HIV-infected persons who are asymptomatic and exhibit low level immunosuppression (CD4 count >200 cells/ $\mathrm{mm}^{3}$ ) can be considered for vaccination, guided by national guidelines. The need for vaccination varies greatly by geographic area and may change over time. For example, a study of $>700$ HIV-infected adults in Austria noted seronegativity of $8.4 \%$ for measles, $33.4 \%$ for mumps, and $18.8 \%$ for rubella; overall, almost half were lacking immunity to one of the components 
and required MMR vaccination [39]. Additionally, a study from Spain noted that nearly 30\% were seronegative to at least one component of the MMR vaccine [40]. In these serostudies, immigrants were at the greatest need for vaccination.

WHO guidelines state that measles vaccination should be routinely administered to potentially susceptible, asymptomatic HIV-positive children and adults. Vaccination can also be considered for those with symptomatic HIV infection if not severely immunosuppressed (i.e., CD4 count $>200$ cells $/ \mathrm{mm}^{3}$ ). Dosing of vaccines are based on national immunization schedules [2].

BHIVA guidelines suggest screening HIV-infected adults for measles IgG regardless of history of childhood vaccination, since this may not be a reliable predictor of current serostatus $[3,41]$. Those who are seronegative and are clinically stable with a CD4 count $>200$ cells/ $\mathrm{mm}^{3}$ should be offered two doses of MMR $>1$ month apart [3]. The EACS recommends avoidance of MMR among those with a CD4 count $<200$ cells $/ \mathrm{mm}^{3}$ (CD4 percent $<14 \%$ ) [8]. For individuals with a CD4 count $>200$ cells/ $\mathrm{mm}^{3}$ and not on ART, vaccination may be delayed until ART is initiated, depending on the likelihood of exposure. Among HIV-infected persons exposed to measles, a screening measles IgG should be obtained regardless of vaccine history; those who are seronegative and meet the above criteria for low-level immunosuppression should receive post-exposure prophylaxis via vaccination within 3 days of contact. Screening for rubella IgG is recommended among HIV-positive women of childbearing age with unknown status; those who are seronegative, have a CD4 count $>200$ cells $/ \mathrm{mm}^{3}$, and are not currently pregnant should be vaccinated with one dose of MMR with a follow-up test for seroconversion, or two doses of MMR vaccine; for those not immune to both rubella and measles, two doses of MMR should be administered [3]. Similar to BHIVA, French guidelines also recommend two doses of MMR among non-immune persons with a CD4 count $>200$ cells $/ \mathrm{mm}^{3}$; vaccination should be avoided during pregnancy, and becoming pregnant should be avoided within the 2 months following vaccination [9]. In addition to pre-exposure vaccination, vaccination within 3 days of measles exposure can be considered among HIV-infected adults without a history of measles or vaccination who have a CD4 count $>200$ cells $/ \mathrm{mm}^{3}$ [9].

In the US, although most ( 85-95\%) HIV-infected adults are immune against measles, studies have shown that $\sim 15 \%$ are seronegative to at least one of the viruses contained in the MMR vaccine, with a higher frequency among younger adults [42-44]; hence, serostatus should be ascertained. The MMR vaccine (two doses at least 28 days apart) can be administered among HIV-infected adults who lack immunity and who have a CD4 count $>200$ cells $/ \mathrm{mm}^{3}$ for at least 6 months $[4,6]$.

Immunologic response rates to vaccination vary by the host's immune status [45]. During the ART era, a study of 26 HIV-infected adults (most receiving ART with a mean CD4 count of 496 cells $/ \mathrm{mm}^{3}$ ) and 22 controls found that measles vaccine responses were not statistically different at 3 months ( $81 \%$ vs. $86 \%$, respectively); at 1 year post-vaccination, a higher proportion of HIV-infected adults had lost measles antibodies; however, cellular responses were similar [46]. A more recent study showed that seroconversion frequencies for measles, mumps, and rubella were $53 \%, 61 \%$, and $83 \%$, respectively, among HIV-infected adults who had a median post-vaccination CD4 count of 526 cells $/ \mathrm{mm}^{3}$ [44]. MMR revaccination among HIV-positive children who did not develop an initial response was effective while on ART $[47,48]$, with a persistence of protection seen at 3 years [49]; however, data are lacking among HIV-infected adults regarding serologic responses to revaccination. Further, there is a lack of data on vaccine responses to the mumps and rubella components of MMR among HIV-infected adults.

Studies have not found adverse immunologic or virologic outcomes after MMR vaccination $[44,50]$. Further, no serious safety issues have been noted when administered to recommended adult groups with CD4 counts $>200$ cells $/ \mathrm{mm}^{3}[45,46]$. The MMRV vaccine should not be administered, since it has not been adequately evaluated in HIV-infected persons and contains seven times more varicella 
antigens than the monovalent varicella vaccine, and hence may pose a safety concern.

\section{TRAVEL-RELATED VACCINES}

HIV-infected travelers may be at increased risk of complications from infectious diseases acquired at their destination [51]. HAV and influenza vaccinations, reviewed in Part I of this series, should be provided based on the individual's itinerary, as these are two of the most commonly acquired infections during travel. HIV-infected persons should carefully consider the benefits and risks of travel to locations with potentially deadly infectious diseases (e.g., traveling to yellow fever endemic locations).

Vaccinations for travelers will be reviewed, and a concise summary of the current recommendations is presented in Table 2. Special attention should be given to expatriates returning to their home countries, since they may falsely believe that they have immunity against endemic infections, but may require vaccinations for protection. Given emerging and changing disease trends, providers are encouraged to review updated resources including those available from national public health programs, the WHO [52], and Yellow Book published by the US Centers from Disease Control and Prevention [53]. In addition to the receipt of recommended vaccinations, travelers should be advised on additional methods to reduce travel-associated infections including avoiding environmental-, arthropod-, and food-related exposures.

\section{Polio}

Poliovirus serotypes 1,2 , and 3 can cause neuroinvasive disease including aseptic meningitis, encephalitis, and a poliomyelitis syndrome characterized by flaccid paralysis. Wild poliovirus type 1 currently circulates in select countries (e.g., Afghanistan, Pakistan, and Nigeria); however, wild-type 2 has been eradicated and type 3 not detected since 2012. There is a paucity of data regarding polio infections among HIV-infected adults, although there are data in infants/children suggesting successful clearance of enteroviruses, including vaccine-related poliovirus $[54,55]$.
While there are two overall types of vaccines against polio [a live oral poliovirus (OPV) and an inactivated polio vaccine (IPV)], the former vaccine (OPV) is contraindicated among immunocompromised hosts since it is a live vaccine and may give rise to vaccine-associated paralytic polio. Additionally, OPV is being phased out globally as part of the final stages of polio eradication.

WHO recommends routine polio vaccination of children based on national guidelines [2]. Travelers to countries where the polio virus is circulating should ensure they have completed the age-appropriate polio vaccine series. In addition, travelers from infected countries should be vaccinated to reduce the international spread of wild poliovirus. Recommendations for HIV-infected persons are similar to those for the general population, and based on national guidelines with the caveat that only IPV should be utilized for vaccination.

BHIVA recommends for the avoidance of OPV in HIV-infected persons and their contacts $[3,56]$, hence IPV is the vaccine of choice. Primary vaccination with a 5-dose course is routinely administered during childhood. BHIVA states that HIV-infected adults requiring vaccination (due to incomplete series or uncertain vaccination history) for diphtheria, tetanus, or polio utilize the Td/IPV vaccine in accordance with national guidelines. Vaccination can be given regardless of CD4 count, HIV viral load, or ART use. A Td/IPV booster is recommended every 10 years to those at risk of exposure, typically through travel. Post-vaccination antibody testing for ensuring a response is not generally recommended, except among those at risk for exposure to poliovirus due to occupation (e.g., laboratory workers). EACS and French guidelines for HIV-infected adults do not specifically mention polio vaccination $[8,9]$.

In the US, beyond the primary vaccine series typically administered during infancy, no further vaccination is recommended except among travelers to endemic areas for polio for which a single lifetime polio booster is recommended using the inactivated form of the polio vaccine (IPV) [4]. Additional vaccination may be required depending on evolving requirements, which should be reviewed prior to departure. 
For example, long-term travelers staying within specific countries (e.g., Afghanistan, Nigeria, and Pakistan) should receive the polio vaccine between 4 weeks and 12 months before departure from the polio-infected countries [57]. Testing for post-vaccination antibody responses among vaccine recipients is not recommended.

Studies regarding immune responses among HIV-infected adults are limited, but one study found a $78-100 \%$ serologic response rate, with the best responses among those with CD4 counts $>300$ cells $/ \mathrm{mm}^{3}$ [58]. Responses to primary or booster doses of polio vaccination may be blunted among patients not receiving ART, and vaccine failures have been described especially among HIV-infected persons with low CD4 counts (e.g., $<200$ cells $/ \mathrm{mm}^{3}$ ) [59]. Data regarding immune responses in children have shown variable results. One study showed a response rate of $>80 \%$ to polio vaccine similar to that of non-infected children [60]. Conversely, another study found significantly lower rates of seroconversion to all three poliovirus serotypes in HIV-infected children compared to noninfected children; however, none of the HIV-infected children were receiving ART and the majority (71\%) were severely immunosuppressed [61]. One study showed good immunogenicity of IPV administered intradermally (compared with intramuscularly) in HIV-infected adults, suggesting this as a potential alternate route for administration, which may assist in making IPV more affordable, especially in developing countries [62]. There is a lack of data on the durability of protection and no current evidence-based recommendations regarding post-vaccination testing or boosters for HIV-infected persons. No specific adverse safety signals have been noted among HIV-infected IPV recipients [58, 63].

\section{Typhoid}

Typhoid fever is an invasive infection caused by Salmonella serotype Typhimurium (or $S$. Typhi), $S$. paratyphi, and other Salmonella species. HIV-infected persons have an increased risk of Salmonella infections including complicated disease with bacteremia and relapsing and/or persistent infection $[64,65]$. The burden of disease has decreased during the ART era, but remains elevated among HIV-infected persons [66].

Vaccines are currently available against $S$. Typhi, but not for other Salmonella species. Both live (i.e., Ty21a given by mouth) and inactivated (e.g., Vi capsular polysaccharide abbreviated as ViCPS given as an injectable) vaccines are available, with the latter recommended among immunosuppressed persons. Protein-conjugated Vi polysaccharide vaccines are licensed in some countries.

WHO recommends for the programmatic use of typhoid vaccines for controlling endemic disease given the high burden of typhoid fever and increasing antibiotic resistance of $S$. Typhi [2]. Vaccination strategies should be based on knowledge of the local epidemiological situation and follow national guidance. In addition to vaccination of residents in high-burden countries, vaccination is recommended for travelers to endemic areas where the risk of typhoid fever is high (especially for those staying in endemic areas for $>1$ month and/or in locations where antibiotic-resistant strains of $S$. Typhi are prevalent). WHO guidelines state that the Vi polysaccharide vaccine (ViCPS) is safe for HIV-infected adults $[1,2]$. Per the guidelines, Ty21a can be administered to HIV-infected adults if they are asymptomatic and the CD4 count is $>200$ cells $/ \mathrm{mm}^{3}[1,2]$.

BHIVA recommends the inactivated ViCPS vaccine for those traveling to areas at risk for $S$. Typhi acquisition, especially those who will be exposed to poor sanitary conditions, long-term stays, and visiting friends/family. Vaccination should be given at least 2 weeks prior to travel to ensure adequate time for developing an immune response prior to exposure. Revaccination is at 3 years among those who remain at risk [3]. While the BHIVA guidelines do not recommend Ty21a (the live vaccine) to be given to HIV-infected persons, the EACS guidelines state that it can be used if the CD4 count is $>200$ cells $/ \mathrm{mm}^{3} \quad$ (CD4 >14\%) [8]. French guidelines do not discuss typhoid vaccination [9].

US guidelines mirror those of the BHIVA [4]. Indications and dosing schedules are the same as for HIV-uninfected persons, except that 
HIV-infected adults should receive ViCPS (Typhim $\mathrm{Vi}^{\circledR}$, Pasteur Merieux) rather than the oral live vaccine. In addition to travelers, persons with intimate exposure (e.g., household contact) to a documented $S$. Typhi chronic carrier and laboratory workers who may be exposed to cultures of $S$. Typhi are recommended for vaccination [67]. Booster doses are to be administered every 2 years for those with continued or repeated exposure.

Lower post-vaccination responses to typhoid vaccination have been described among HIV-infected persons, and poorer responses are correlated with low CD4 counts ( $<200$ cells/ $\mathrm{mm}^{3}$ ) [68]. Data on the durability of immune responses are lacking among HIV-infected adults and recommendations regarding the timing of repeat doses among those who remain at risk are the same as for the general population. Similar to other travel-related infections, HIV-infected patients should be counseled that vaccination may not offer complete protection and be advised regarding additional preventive measures (e.g., avoidance of potentially contaminated food and water).

There are no known significant safety issues with the use of the inactivated typhoid vaccine among HIV-infected persons. A study that reviewed the safety live oral vaccines, including the typhoid vaccine, in 34 HIV-infected adults found no major adverse events except for late episodes of diarrhea, which may not be exclusively due to vaccination [69]. Since Ty21a is a live-attenuated vaccine, antibacterials and certain antimalarials (e.g., proguanil, mefloquine) may interfere with vaccine efficacy and should be discontinued 3 days prior through 3 days post-administration [52].

\section{Yellow Fever}

Yellow fever is a flavivirus infection transmitted by Aedes mosquitos and found in tropical and sub-tropical areas of South America and Africa. It is unknown whether HIV-infected persons have a higher risk for acquisition or more severe illness due to yellow fever.

WHO recommends yellow fever vaccination for three overarching reasons: to protect populations living in areas of disease; to protect travelers visiting these areas; and to prevent international spread by minimizing the risk of importation of the virus by viremic travelers [2]. WHO guidelines state that yellow fever vaccination may be offered to asymptomatic HIV-infected adults with CD4 counts $\geq 200$ cells $/ \mathrm{mm}^{3}$. For persons with symptomatic HIV or with CD4 counts $<200$ cells $/ \mathrm{mm}^{3}$, vaccination should not be administered [2]. The WHO advocates studies to identify risk groups (such as HIV-infected patients) who may benefit from booster doses of vaccine and research on the durability of immune responses among these groups.

BHIVA recommends that HIV-infected adults aged $<60$ years with a CD4 count $>200$ cells/ $\mathrm{mm}^{3}$ who are traveling to a country with risk of yellow fever exposure be offered the choice of vaccination. Patients should be counseled regarding the benefits and risks of vaccination in relation to the risk of exposure [3]. If the trip does not truly have a risk for exposure (but vaccination is being considered based on international travel requirements), then a waiver or exemption letter should be considered rather than vaccination (of note, some countries may not accept waivers). Both EACS and French guidelines state a CD 4 count $<200$ cells $/ \mathrm{mm}^{3}$ is a contraindication for vaccination $[8,9]$. Yellow fever vaccination should be given at least 2 weeks prior to travel. Although data among immunocompetent hosts suggest that vaccine durability exceeds 10 years (hence new guidelines advocate for a lifetime dose rather than booster doses every 10 years), data are lacking among HIV-infected adults, hence a booster every 10 years is advised among those at continued risk if the patient remains $<60$ years of age and with a CD4 count $>200$ cells $/ \mathrm{mm}^{3}$ [3] The guidelines also suggest considering serologic testing prior to revaccination to assess the need for further vaccine doses, especially among those at greater risk for vaccine-related side effects. HIV-infected persons who are $>60$ years, have a CD4 count $<200$ cells $/ \mathrm{mm}^{3}$, or are pregnant should not receive yellow fever vaccination per BHIVA guidelines [3].

US guidelines recommend that HIV-infected persons should ideally avoid traveling to yellow fever endemic locations. Among those who 
cannot alter their itineraries and who will be at risk of infection, vaccination can be considered for those with asymptomatic HIV infection and a CD4 count $>200$ cells $/ \mathrm{mm}^{3}$. Specifically, yellow fever vaccination is a precaution for asymptomatic HIV-infected persons with CD4 cell counts of $200-499$ cells $/ \mathrm{mm}^{3}$, hence the benefits and risks should be carefully discussed. Vaccination is not a precaution for people with asymptomatic HIV infection and CD4 cell counts $\geq 500$ cells $/ \mathrm{mm}^{3}$ and can be administered if recommended. Yellow fever vaccination is contraindicated among those with a CD4 counts $<200$ cells $/ \mathrm{mm}^{3}$ or those with symptomatic HIV infection [70]. Close attention to the itinerary is suggested to ensure that vaccination is truly needed [51, 71]. If international travel requirements and not true exposure risk are the only reasons to vaccinate a traveler, a waiver letter should be provided (travelers should be warned that vaccination waiver documents might not be accepted by some countries). Travelers should be informed that immune responses to vaccination may be muted and hence serologic testing (available through public health departments and the CDC) be considered and performed 1 month after vaccination. Guidelines suggest that those who were infected with HIV when they received their last dose of yellow fever vaccine should receive a dose every 10 years if they continue to be at risk.

The best option for HIV-infected travelers with low CD4 counts (e.g., $<200$ cells $/ \mathrm{mm}^{3}$ ) is cancelling travel to the endemic location. If the patient is unwilling to alter their itinerary, obtaining a waiver and adhering to strict mosquito avoidance can be considered, but carries the risk for acquiring yellow fever.

Regarding immune responses to yellow fever vaccination, HIV patients have lower vaccine response rates compared to HIV-uninfected persons based on studies including a Cochrane review [72-77]. One study showed that 83\% developed neutralizing antibody compared with $97 \%$ of HIV-uninfected persons $(p=0.01)$ [78]. The immune response is greater among those with higher CD4 counts and lower HIV viral loads [77, 78]. Another study found that 98\% of HIV-infected persons had an initial seroresponse, which decreased to $92 \%$ at 10 years post-vaccination with durability being closely related to suppressed HIV viral loads over time [77].

A Cochrane review states that yellow fever vaccine may be potentially safe in HIV-infected patients, although conclusions are limited by the small numbers of reported patients receiving vaccination [72]. Specifically, an evaluation of $\sim 500 \mathrm{HIV}$-infected persons meeting vaccine guidelines found no severe adverse events among recipients; however, these data do not exclude infrequent adverse events [72]. Severe adverse events have been described among HIV-infected persons with low CD4 counts, including a case of post-vaccination myeloencephalitis in a person with a CD4 count of 108 cells $/ \mathrm{mm}^{3}$ [79]; no cases of vaccine-related viscerotropic disease in HIV patients have been described to date [51]. Risk for severe adverse events post-vaccination include age $>60$ years, immunosuppression, and thymus disorder, which are contraindications to vaccination. Less severe vaccine-related adverse events may include headache, myalgias, fever, and local site reactions which have been noted in $10-30 \%$ of recipients.

\section{OTHER TRAVEL-RELATED VACCINES}

HIV patients may require additional vaccinations prior to travel or for those living in endemic areas. These may include rabies, Japanese encephalitis virus (JEV), cholera, and tick-borne encephalitis (TBE) vaccines. There are few specific data regarding these vaccines among HIV patients, hence guidance generally follows standard travel guidelines with some important caveats as noted below.

Some guidelines (e.g., US) note that, since vaccines given to protect against travel-related diseases among HIV-infected persons with a CD4 count $<200$ cells $/ \mathrm{mm}^{3}$ are often suboptimal, revaccination $\geq 3$ months after immune reconstitution on antiretroviral therapy can be considered. Additionally, delaying travel pending reconstitution of the CD4 count on antiretroviral therapy should be considered to 
bolster vaccine responses and minimize the risk of infection [80].

\section{Rabies}

Rabies is a virus in the Lyssavirus genus that can be transmitted to humans by the bite or scratch of a rabid animal (most commonly dogs and bats, but also from a wide range of animals) resulting in encephalomyelitis that is typically fatal. Rabies is present on every continent except Antarctica, and public health guidance should be sought regarding the risk of rabies after potential exposure by geographic area and animal type. HIV-infected persons appear to be at similar risk for rabies with no known differences in the disease natural history in this group.

Rabies vaccines include the human diploid cell vaccine (HDCV), the purified chick embryo cell vaccines (PCECV), and the purified Vero cell rabies vaccine (PVRV). These vaccines are inactivated, and may be used as pre- or post-exposure prophylaxis.

WHO recommends a 3-dose vaccination series [concentrated and purified cell-culture (CCV) or embryonated egg-based (EEV) rabies vaccines, jointly referred to as CCEEVs] for pre-exposure prophylaxis, but does not specifically provide additional guidance for HIV-infected adults [2]. For post-exposure prophylaxis, a complete series of five doses of intramuscular vaccines (CCEEVs) is recommended along with human rabies immunoglobulin (HRIG) among previously unvaccinated persons. Rabies virus neutralizing antibody testing is recommended at 2-4 weeks following vaccination to assess the possible need for an additional dose of the vaccine among immunosuppressed persons.

BHIVA guidelines recommend pre-exposure vaccination of HIV-infected adults who are risk of rabies exposure due to travel using three doses of a cell culture-derived vaccine (HDCV and PCECV are available in the UK) at days 0,7 , and 28. Those at risk for a poor vaccine response (based on CD4 count, HIV viral load, and lack of ART use) should be offered post-vaccination rabies serology testing at 2-4 weeks after the 3rd vaccine dose, and those with a level $<0.5 \mathrm{IU} / \mathrm{mL}$ offered a booster dose (which can be given as a double dose) followed by repeat serology. Antibody testing in the UK can be obtained through the PHE Rabies clerk at +44 (0) 2083276204 [3]. For persons who remain at risk for rabies through ongoing travel, a booster is recommended at 1 year after the initial completion of the series, with subsequent boosters every 3-5 years or based on serology test results when indicated [3]. HIV-infected persons at risk of rabies through occupation (e.g., laboratory workers, veterinary staff, etc.) can be offered the 3-dose vaccine series and follow-up testing/boosters if they have a CD4 count $>200$ cells $/ \mathrm{mm}^{3}$ and stable HIV viral load suppression. For those at continuous risk, follow-up serologies are recommended every 6 months (and booster doses among those with lack of seroprotection) and, for those with lower exposure risks, monitoring of serologic testing is advised every 1-3 years. Those who do not mount an adequate vaccine response and those with a CD 4 count $<200$ cells $/ \mathrm{mm}^{3}$ (given poor vaccine response rates) should avoid exposure [3]. BHIVA also offers post-exposure vaccination guidance and recommends a 5-dose series $(0,3$, 7, 14 and 30 days) along with HRIG. Those who had received pre-exposure vaccination with a documented adequate post-vaccination response and ongoing HIV control (i.e., CD4 count $>500$ cells $/ \mathrm{mm}^{3}$, HIV viral load suppression, and receipt of ART) at the time of vaccination and thereafter, should receive two vaccine doses ( 0 and 3-7 days). All persons should have post-vaccination antibody serology testing 2 weeks after the last vaccine, and non-responders should be offered double doses of the vaccine and consideration for combined intradermal and subcutaneous routes of administration [3]. EACS and French guidelines do not discuss rabies vaccination $[8,9]$.

US guidelines recommend vaccination among HIV-infected individuals using the same criteria as for HIV-uninfected persons [4]. In brief, a pre-exposure vaccination series of three doses is recommended for those at risk of exposure with post-vaccination serologic testing at 6 months for those at continuous risk and 2 years for those at frequent risk, with a booster dose if antibody titer is below acceptable level. 
For post-exposure prophylaxis among those without pre-exposure vaccination, the recommendation is a 5 -dose vaccine series at $0,3,7$, 14 , and 28 days (the 5 th dose is added for HIV-infected and other immunocompromised persons vs. the 4-dose series for immunocompetent persons) and HRIG. Previously vaccinated persons who received one of the ACIP-recommended pre-exposure prophylaxis regimens with cell-culture vaccines, or those who received another vaccine regimen with documented adequate rabies virus-neutralizing antibody response, should receive post-exposure prophylaxis with two vaccine doses $(0$, 3 days). When rabies pre- or post-exposure prophylaxis is administered to an immunosuppressed person (including those with HIV), serum samples should be tested for rabies virus neutralizing antibody to ensure that an acceptable antibody response has developed after completing the series. Interpretation of the results and laboratories performing this testing in the US can be found at: http://www.cdc.gov/ rabies/specific_groups/doctors/serology.html. If an acceptable antibody response is not detected after the final dose in the pre- or post-exposure prophylaxis series, guidelines recommend consultation with their physicians and appropriate public health officials. Durability of responses and frequency of repeat antibody testing is not addressed in current US guidelines.

Vaccine immunogenicity may be reduced among HIV-infected persons, especially among those with low CD4 counts, high HIV viral loads, and lack of ART use [81-87]. In one study, $88 \%$ of those with a CD 4 count $>450$ cells $/ \mathrm{mm}^{3}$ on ART developed a protective response after three doses of the cell culture-based vaccine [87]. Since responses may vary and persons with even high CD4 counts ( $>500$ cells $/ \mathrm{mm}^{3}$ ) may not develop protective responses, post-vaccination serology should be considered [3]. In addition to the initial response, immune responses may wane faster particularly among those not receiving ART. For example, a study evaluating a two-dose rabies vaccine regimen in 30 HIV-infected adults (median CD4 count of 537 cells $/ \mathrm{mm}^{3}$ ), found that 5 years after primary vaccination, $63 \%$ had antibody titers above the protection threshold compared with $86 \%$ of healthy controls [86]. Thus, follow-up testing among those who remain at risk should be considered [3, 86, 87]. Studies on the utility of double vaccine doses and the potential use of intradermal (vs. intramuscular) routes of administration for bolstering vaccine responses among HIV-infected persons are needed [88].

Vaccinations are typically well tolerated among HIV-infected persons with injection site reactions and mild systemic reactions most commonly observed [82, 83, 86, 88].

\section{Japanese Encephalitis Virus}

JEV is a flavivirus transmitted by Culex mosquito bites. The disease is found in Asia, most commonly in the Southeast and Western Pacific areas; rural areas with rice fields and pig farms are associated with the highest risk. Seasonal patterns exist within individual countries (e.g., in temperate areas of Asia, transmission usually peaks in summer and fall) and yearly based on rainfall (e.g., the highest transmission rates are during and just after the wet seasons). Infection may range from asymptomatic to life-threatening encephalitis. Whether HIV-infected persons have a higher risk for infection or more severe disease is unknown.

There are a variety of inactivated JEV vaccines available including the Vero cell-derived vaccine $\left(\mathrm{IXIARO}^{\circledR}\right)$. A previous mouse brain-derived vaccine is no longer recommended given its adverse safety profile compared with other vaccine preparations.

WHO recommends JEV vaccination be integrated into national immunization schedules in areas where JEV is a public health priority [2]. Guidelines advise that JEV vaccine can be used in immunocompromised persons, including HIV-infected individuals, but that the immune response may be lower than in fully immunocompetent persons, and that inactivated Vero cell-derived vaccines should be used preferentially over live attenuated or live recombinant vaccines. A booster dose is recommended to be given at $>1$ year after primary series if there is continued risk [2].

BHIVA recommends JEV vaccination among those at risk based on travel or occupation with an 
inactivated Vero cell-derived vaccine (e.g., IXIARO $^{\circledR}$ ) using the same schedule as for the general population (two doses given 24-28 days apart) [3]. In general, vaccination is recommended for travelers to an endemic area staying for $>1$ month during the transmission season, especially when travel involves rural areas and/or pig farms. An abbreviated schedule is generally not recommended for HIV-infected persons unless there is an urgent need for completion of the series prior to exposure. A booster vaccine is recommended at 12-24 months among those at continued risk, with another booster at 10 years [3].

US guidelines note the lack of safety or efficacy data regarding JEV vaccines among immunocompromised persons. When utilized, the inactivated vaccine (Vero cell-derived vaccine, IXIARO ${ }^{\circledR}$ ) at the usual dose and schedule is advised. Vaccination is recommended for travelers who plan to spend $\geq 1$ month in endemic areas during the JEV transmission season [4].

The immunogenicity and clinical efficacy of JEV vaccination in HIV-infected adults are unknown. Data among HIV-infected children suggest reduced antibody responses measured by geometric mean titers, but that most develop seroprotective responses with the best responses among those on ART [89-92].

Vaccine safety appears comparable among HIV-infected and HIV-uninfected persons based on limited pediatric data [92].

\section{Cholera}

$V$. cholerae is a Gram-negative bacterial infection acquired after consuming contaminated water or food with clinical manifestations of acute, profuse watery diarrhea. In its extreme manifestation, cholera is one of the most rapidly fatal infectious illnesses known. Serogrouping is based on polysaccharides of the somatic (O) antigen with more than 200 serogroups. Two serogroups-O1 and O139-cause epidemic disease with $\mathrm{O} 1$ causing $>99 \%$ of global cases. Regarding cholera in HIV-infected persons, limited data suggest that that this group has an increased risk for severe disease [93].

There are several inactivated, oral cholera vaccines available worldwide which are described below. In addition, a live attenuated vaccine (CVD 103-HgR) is also available in some countries.

WHO recommends vaccination in cholera-endemic countries targeting high-risk areas and population groups [2]. The primary groups for vaccination in endemic areas are preschool-aged and school-aged children, but other groups for targeting include pregnant women and HIV-infected individuals who are at risk for severe disease. The two main vaccines recommended by the WHO include Dukoral ${ }^{\circledR}$ [a killed whole cell (WC) vaccine with cholera toxin B subunit (WC-rBS)] and Shanchol ${ }^{\circledR}$ [a modified killed whole cell-only vaccine (WC-only)]. Shanchol ${ }^{\circledR}$ differs from Dukoral ${ }^{\circledR}$, in that it also covers serotype O139. Dosing for Durkoral $^{\circledR}$ is by two oral doses given $\geq 7$ days apart (but $<6$ weeks apart) and Shanchol ${ }^{\circledR}$ as two oral doses given 14 days apart. Booster doses are every 2nd year among adults who remain at risk; if the timing of the booster is $>2$ years for Dukoral ${ }^{\circledR}$, then repeating the primary 2-dose vaccine series is advised.

BHIVA recommends that HIV-infected adults at significant risk for cholera due to their travel itinerary be vaccinated regardless of CD4 count, HIV viral load, or ART receipt [3]. The oral, non-replicating vaccine, WC/rBS (Dukoral ${ }^{\circledR}$ ), is recommended and should be given at least 1 week prior to potential exposure with a booster dose after 2 years if continued protection is needed [3].

In the US, a live attenuated CVD 103-HgR vaccine, Vaxchora $^{\mathrm{TM}}$, has recently been approved as a single-dose oral cholera vaccine for adults aged 18-64 years traveling to an area of active toxigenic $V$. cholerae $\mathrm{O} 1$ transmission. Specifically, travelers with high risk of exposure to cholera or a higher risk for poor outcomes should be vaccinated. Travelers at high risk for cholera include those traveling to provide humanitarian aid in cholera outbreak settings, visiting friends and relatives, staying for a long time, or with limited access to safe food and water [4]. US guidelines do not specifically discuss HIV-infected adults. The WHO prequalified vaccines (e.g., Dukoral ${ }^{\circledR}$ and Shan$\mathrm{Col}^{\mathbb{B}}$ ) are not available in the US.

The clinical efficacy of cholera vaccination has not been extensively studied in 
HIV-infected persons, but has a rate of $65-86 \%$ in the general population [3]; a single study in Africa which included $~ 25 \%$ HIV-infected persons noted an efficacy of $72 \%$ [94]. Immunogenicity data among HIV-infected persons suggest good, albeit diminished, immune responses after two vaccine doses, with the best responses among those with higher CD4 counts $[95,96]$. In a study of Vaxchora ${ }^{\mathrm{TM}}$, serological responses were significantly diminished among the HIV-infected group, especially in those with CD4 counts $<500$ cells $/ \mathrm{mm}^{3}$ [97]. Most studies have been conducted among persons from endemic areas which may have influenced vaccine responses, and there is currently a lack of data on vaccine responses among HIV-infected travelers from non-endemic areas. Further, the duration of protection of vaccines beyond 3-6 months is currently unknown among HIV-infected persons.

Regarding safety, the aforementioned killed vaccines have been well tolerated among HIV-infected persons to date [94, 95, 97, 98]; adverse reactions are uncommon but may include fatigue, headache, abdominal pain, nausea, vomiting, diarrhea, and lack of appetite. The safety of Vaxchora ${ }^{\mathrm{TM}}$ has not been evaluated in the immunocompromised population; however, a previously licensed Orochol vaccine, which also contains the CVD 103-HgR strain used in Vaxchora $^{\mathrm{TM}}$, was found to be safe in HIV-infected adults [97].

\section{Tick-Borne Encephalitis}

TBE virus is a Flavivirus transmitted primary by Ixodes tick bites and is the most common tick-transmitted disease in Central and Eastern Europe and Russia. TBE includes three subtypes: European, Siberian, and Far Eastern. Approximately two-thirds of cases are asymptomatic, with one-third developing symptoms approximately 8 days post-infection, ranging from a mild febrile illness to severe encephalomyelitis; the Far Eastern subtype typically is associated with more severe disease, and the European subtype with milder disease. The estimated risk for acquiring TBE in an unvaccinated traveler to a highly endemic area is $1 / 10,000$ [99]. It is unknown if HIV infection increases the risk for either infection or disease severity. There are data to suggest that deletion in the chemokine receptor 5 (CCR5) gene (i.e., being homozygous for CCR5Delta32) is associated with increased risk and severity of TBE [100]; whether the use of the CCR5-blocker, maraviroc, also increases the risk is unclear at this time.

Currently, there are four widely used TBE vaccines: FSME-Immun ${ }^{\circledR}$ and Encepur ${ }^{\circledR}$ (manufactured in Europe), and TBE vaccine Moscow ${ }^{\circledR}$ and EnceVir $^{\circledR}$ (manufactured in the Russian Federation) [2]. FSME-Immun ${ }^{\circledR}$ is also licensed in Canada, but there are no TBE vaccines currently licensed or available in the US. There is a vaccine produced in China against TBE, but limited data exist. The dosing schedule for each vaccine varies, and should follow manufacturer and national guidelines. Immunogenicity studies suggest that the aforementioned European and Russian vaccines should provide cross-protection against all subtypes.

WHO recommends vaccine guidance based on national and local-level risk disease assessments. In areas where disease is highly endemic ( $>5$ cases $/ 100,000$ per year), WHO recommends vaccination among all ages in which the vaccine is approved and makes special note for vaccinating those $>50$ years given their increased risk for severe disease; there is no specific mention regarding vaccination of HIV-infected persons [2]. Among areas of low incidence, local guidance should be followed and target those most likely to be affected. Finally, travelers from non-endemic to endemic areas with planned extensive outdoor activities should be offered vaccination. The standard vaccination schedule includes three doses in the primary series, followed by boosters guided by national guidelines; for those at risk for more severe disease (e.g., $>50$ years), boosters are advised at $3-5$ years. Vaccination for post-exposure prophylaxis after a tick bite is not recommended given that the time to develop an immune response would likely not prevent infection.

BHIVA recommends that HIV-infected adult travelers who may be exposed to TBE during the seasons of disease (i.e., typical transmission seasons in Europe are May/June and then again in September/October) be offered vaccination 
[3]. Among those residing in endemic areas, the guideline recommends following local vaccination program guidance. Vaccination series is typically with three doses; however, BHIVA guidelines recommend a 4 -dose series $(0,1,2$, and 9-12 months) for HIV-infected adults given concerns for lower vaccine responses in this group [3]. An accelerated vaccine schedule exists ( 0 and 2 weeks, then a 3rd dose at 5-12 months), but no data are available on its immunogenicity among HIV-infected persons, hence the urgency of vaccination should be weighed against potential poorer immune responses. Boosters are recommended at 3-5 years, with the shorter interval among those with CD 4 counts $<400$ cells $/ \mathrm{mm}^{3}$. Boosters can be guided by serological test results with a level $>126$ Vienna units/mL considered as protective [3].

US guidelines advise consideration of vaccination among travelers with anticipated high-risk exposures (working or camping in forested areas or living in TBE endemic countries for extended periods of time). However, given the lack of vaccines in the US, vaccination would need to occur outside that country (e.g., Canada or in the country of travel); however, the multi-dose vaccine series makes this potentially challenging [53]. No specific guidance for HIV-infected persons is provided.

The clinical efficacy of TBE vaccination among HIV-infected persons is unstudied. Regarding immunogenicity, HIV patients (especially those with a CD 4 count $<400$ cells/ $\mathrm{mm}^{3}$ ) have poorer responses compared with HIV-uninfected persons [101, 102], with vaccine durability unknown.

TBE vaccines are overall safe and most commonly involve local site reactions, but data are limited among HIV-infected persons. Vaccination is contraindicated in those with severe egg allergy.

\section{OCCUPATIONAL-RELATED VACCINES}

Occupational-related vaccines remain an important consideration given the ongoing threat of bioterrorism. Although there are limited data on the use of vaccines against anthrax and smallpox in HIV-infected persons, given the large global population of HIV-infected persons (36.7 million as of 2015), and that HIV-infected persons work in a wide variety of occupations, including in the military, these specific vaccinations are reviewed. There are other vaccinations that may be recommended depending on specific occupational risk and location of residence (e.g., Q-fever vaccine available in Australia; adenovirus vaccine in the US military recruit population), hence local/national guidance should be consulted.

\section{Anthrax}

Bacillus anthracis is a toxin-producing bacterial infection transmitted via contact with infected animals or their products (e.g., furs). The three forms of the disease are cutaneous, respiratory, and gastrointestinal. Infection may become disseminated with bacteremia and meningitis. Most cases occur in Africa, Asia, the Americas, and occasionally in parts of Europe. It is unknown if HIV-infected persons have an altered natural history of disease or higher mortality.

The WHO does not have specific guidelines on the use of anthrax vaccination among HIV-infected adults.

BHIVA recommends pre-exposure vaccination of HIV-infected adults who are at significant risk for anthrax exposure (e.g., based on occupation) using national guidelines [3]. Vaccination can be given regardless of CD4 count, HIV viral load, or ART use. The Anthrax Vaccine Precipitated (AVP) is the recommended vaccine and is a non-replicating cell-free filtrate of $B$. anthracis proteins. The vaccine is available in the UK through the Department of Health or Ministry of Defence and given as four doses (0, 3,6 weeks, and 6 months) with a booster given annually among those at ongoing risk. Those with a low CD 4 count $\left(<200\right.$ cells $\left./ \mathrm{mm}^{3}\right)$ may have poorer vaccine responses, and should be counseled on the possibility of lack of protection; additionally, vaccination may be deferred or repeat vaccination considered after immune reconstitution based on the circumstances regarding exposure. Post-exposure vaccination 
(along with receipt of antibiotics) is based on standard recommendations [3].

The US does not have specific guidelines for vaccination of HIV-infected persons. The US CDC recommends pre-exposure vaccination for three groups of adults 18-65 years of age who may be at risk for occupational exposure: certain laboratory workers who work with anthrax, people who handle animals or animal products that may be infected (e.g., veterinarians), and certain US military personnel. In the US, the anthrax vaccine $\left(\right.$ BioThrax $\left.^{\circledR}\right)$ is made from cell-free filtrates of an avirulent, nonencapsulated strain of $B$. anthracis. The vaccine regimen consists of five intramuscular doses (at 0, 1, 6, 12, and 18 months), followed by annual boosters thereafter. For post-exposure treatment, the CDC recommends three subcutaneous doses (at 0,2 , and 4 weeks) of the anthrax vaccine for previously unvaccinated persons $\geq 18$ years who are exposed to aerosolized $B$. anthracis spores, as well as 60 days of appropriate antimicrobial prophylaxis [103].

The efficacy and immunogenicity of anthrax vaccines among HIV-infected persons are unknown. The effectiveness of the anthrax vaccine has been estimated as $93 \%$ for immunocompetent persons who complete the primary series and maintain booster doses. The vaccine is considered safe in the general population, although local site reactions are common ( 50\%); its safety among HIV-infected persons is unknown.

\section{Smallpox}

Variola virus causes smallpox and is acquired through droplets or aerosol typically from an infected person. Smallpox was declared eradicated in 1980 and routine vaccination was stopped. Given concerns for its use in bioterrorism, vaccination was restarted in some countries among targeted groups including military personnel and healthcare workers.

There are three main smallpox vaccines available: ACAM $2000^{\circledR}$, which is a live, replicating vaccinia virus vaccine; Aventis Pasteur Smallpox Vaccine (APSV) which is a replication-competent vaccinia investigational vaccine; and Modified Vaccinia Ankara (MVA,
Imvamune ${ }^{\circledR}$ in the United States and Canada, or Imvanex ${ }^{\circledR}$ in Europe) which is a live, attenuated vaccine that is replication-restricted after multiple cell culture passages. MVA was approved under exceptional circumstances due to the lack of clinical data, but due to a desire for a non-replicating vaccine to be available for potential use in immunosuppressed persons. While guidelines generally recommend avoiding ACAM2000 ${ }^{\circledR}$ among immunosuppressed persons (e.g., HIV-infected persons), in the setting of post-exposure prophylaxis, it can be considered since the data for the efficacy of MVA in preventing smallpox is less certain, and it requires two doses to be administered 4 weeks apart to achieve an immune response comparable to that of the replication-competent smallpox vaccines $[104,105]$.

The WHO does not provide current guidelines on the use of smallpox vaccination among HIV-infected adults.

BHIVA recommends a careful appraisal of the risk of contracting smallpox versus possible vaccine-related adverse events. In cases that pre-exposure vaccination is recommended, but there is no urgency, HIV-infected adults should be given Imvanex $^{\circledR}$ (non-replicating vaccine) with two doses administered at least 1 month apart. No current data exist on booster doses after Imvanex ${ }^{\circledR}$ vaccination. In cases where there is a significant risk for exposure and there is urgency for protection, ACAM $2000^{\circledR}$ can be considered among HIV-infected persons with CD4 counts $>200$ cells $/ \mathrm{mm}^{3}$ after careful consideration of the potential risks. Primary ACAM $2000^{\circledR}$ vaccination is typically with a single dose followed by a booster at 3 years if the risk for exposure remains; no data for alternate dosing strategies exist for HIV-infected persons. Among those exposed to smallpox virus, BHIVA recommends that those with CD4 counts $>50$ cells $/ \mathrm{mm}^{3}$ can be considered for ACAM $2000^{\circledR}$, with the goal for timing of vaccination within 3 days of exposure (but can be given up to 10 days). For those with a CD4 count $<50$ cells $/ \mathrm{mm}^{3}$, BHIVA recommends antiviral medications and possible Invanex $^{\circledR}$ vaccination, but expert opinion is advised. HIV-infected persons should avoid close contact with recipients having received ACAM $2000^{\circledR}$ to avoid possible exposure [3]. 
US guidelines state that HIV-infected adults with CD4 cell counts $\geq 200$ cells $/ \mathrm{mm}^{3}$ who are at high risk for smallpox infection, but without known exposure, can receive pre-exposure vaccination with ACAM $2000^{\circledR}$. On the other hand, persons with CD4 cell counts of 50-199 cells/ $\mathrm{mm}^{3}$ who are at high risk for smallpox infection, but without known exposure, are to be vaccinated with Imvamune ${ }^{\circledR}$ [105]. Among HIV-infected persons who are exposed to smallpox virus, post-exposure vaccination with ACAM $2000^{\circledR}$ is recommended among those with a CD4 count $\geq 50$ cells $/ \mathrm{mm}^{3}$, but avoided among those with a CD4 count $<50$ cells $/ \mathrm{mm}^{3}$, given their higher likelihood of poor immune responses and an increased risk for adverse events, which mirrors guidance from BHIVA [105]. Among this group, if antivirals are not immediately available, it is reasonable to consider the use of Imvamune ${ }^{\circledR}$.

The efficacy of ACAM $2000^{\circledR}$ among HIV-infected adults is unknown. This vaccine was inadvertently administered to ten HIV-positive military members (mean CD4 count 483 cells/ $\mathrm{mm}^{3}$, range 286-751) with noted robust immune responses and no reported adverse events [106]. However, adverse events including progressive vaccinia have been reported in HIV-infected persons with low CD4 counts [107]. Further, ACAM $2000^{\circledR}$ may cause a variety of complications including myopericarditis, encephalitis, generalized vaccinia, eczema vaccinatum, ocular complications including blindness, and death.

Regarding MVA, it was studied in 91 HIV-infected persons with CD4 counts $\geq 350$ cells $/ \mathrm{mm}^{3}$ and HIV viral loads $<400$ copies/mL with antibody responses comparable between HIV-infected and HIV-uninfected persons with a good safety profile [108]. A smaller study $(n=10)$ found similar vaccinia-specific humoral immune responses among HIV-infected individuals and healthy subjects with responses maintained over 1 year [109]. Most recently, a modified vaccinia Ankara-Bavarian Nordic (MVA-BN) vaccine (two doses given 4 weeks apart) was studied in vaccinia-naive $(n=351)$ and vaccinia-experienced $(n=131)$ HIV-infected persons (CD4 count $\geq 200$ cells $/ \mathrm{mm}^{3}$ ) with data suggesting it was safe and immunogenic [110]. The most common side effect of non-replicating smallpox vaccines is injection site reactions; no increased risk for myopericarditis has been noted.

\section{ADDITIONAL VACCINES}

\section{Haemophilus influenzae}

Haemophilus influenzae includes both non-encapsulated and encapsulated strains, the latter including serotype B (Hib) which is a cause of respiratory infections and meningitis. Invasive $H$. influenzae serotype B infections used to be more common among HIV-infected persons; however, more recently, the absolute risk of disease has fallen after Hib vaccination programs [111]. Further, most infections among HIV-infected persons are now due to non-Hib strains, hence the current vaccine offers no protection [112].

An $H$. influenzae type b (Hib) conjugate vaccine is available as an inactivated, capsular polysaccharide conjugated to a protein that protects against invasive serotype $\mathrm{B}$, but not against other serotypes.

WHO guidelines recommend for the inclusion of conjugate Hib vaccines in all infant immunization programs and notes that HIV-infected children should specifically be vaccinated given their higher risk for disease [2]. Guidelines do not mention the vaccination of HIV-infected adults.

BHIVA does not routinely recommend Hib vaccination among HIV-infected adults. If the patient has risk factors for severe Hib disease such as asplenia or complement deficiency (including use of a complement inhibitor therapy), then a single dose of vaccine (e.g., Hib/MenC) is given regardless of prior vaccination or HIV control [3]. EACS does not have specific recommendations [8], and French guidelines recommend its use in children per guidelines for the general population and for adults with particular concurrent conditions such as asplenia [9].

US guidelines offer similar guidance as the BHIVA and no longer recommend Hib vaccination based solely on HIV-positive status. 
Adults who have anatomical or functional asplenia or sickle cell disease should receive one dose of Hib if they have not previously received the vaccine [4].

Regarding immunogenicity, HIV-infected adults can mount protective antibodies, although these are typically lower than among HIV-uninfected persons and are related to the CD4 count [113-115]. There are no additional safety concerns regarding vaccination among HIV-infected adults.

\section{BCG}

The Mycobacterium tuberculosis complex includes M. tuberculosis, M. bovis, and M. africanum. The disease is spread person-to-person through airborne droplets and causes pulmonary and/or extrapulmonary infection. HIV infection increases the risk for reactivation of latent infection and the development of active disease [116]. Tuberculosis (TB) is the leading cause of death among HIV-infected persons in many places in the world including sub-Saharan Africa [117].

A BCG vaccine containing a strain of $M$. bovis is available as a live, attenuated vaccine. While this vaccine may protect against disseminated TB (e.g., meningitis) in children, its efficacy varies widely [118].

The WHO, French, and US guidelines all state that the use of BCG vaccination is contraindicated among persons known to be positive for HIV infection [1, 2, 4, 9]. The BHIVA guidelines state that the BCG vaccine is absolutely contraindicated among HIV-infected persons regardless of CD4 count, HIV viral load, clinical status, or ART use [3]. The avoidance of vaccination among HIV-infected persons is due to safety concerns. Additionally, the efficacy of BCG vaccination among HIV-infected persons is unknown [3]. The mainstay for TB prevention is avoidance of contact with persons or settings that may result in TB exposure. Regular screening for latent TB and, if positive, administration of preventive therapy are advised.

There are notable safety concerns with the use of BCG vaccination among HIV-infected persons given their heightened risk for developing disseminated disease (e.g., osteitis, adenitis) due to the vaccine strain post-vaccination [119-122].
Fatalities have been described and complications may occur several years to decades after vaccination [123-126]. Hence, BCG vaccine is not advised by any of the guidelines for use among HIV-infected adults.

\section{FUTURE DIRECTIONS}

Vaccines are available to protect HIV-infected adults worldwide against an increasing number of infectious diseases, and several novel vaccines are on the horizon (e.g., against dengue, Chikungunya, Ebola, herpes simplex 2, Zika, etc.). Current challenges include ensuring vaccination coverage among HIV-infected persons worldwide. Obstacles are many and may include cost and availability of the various vaccines in developing countries, as well as insurance coverage requirements (e.g., that require that vaccines are given by a general practitioner vs. specialist) and documentation of vaccinations over time in the developed world. The ideal timing of vaccinations also remains an important consideration, including whether to wait until after ART initiation and the optimal CD4 count and HIV RNA level at the time of vaccination. Additionally, studies are needed to address the clinical efficacy and durability of immune responses post-vaccination among HIV-infected adults, and whether HIV patients require alternate dosing or booster schedules for many of the vaccines discussed in this review. Further, data on the need and timing of antibody levels post-vaccination and their correlation with vaccine efficacy among HIV-infected are needed. Finally, for vaccines, especially those administered routinely in childhood/adolescence in many countries (e.g., HPV, hepatitis A and $\mathrm{B}$, and conjugate pneumococcal), the need for boosters during adulthood among HIV-infected persons is a key area for future research.

\section{SUMMARY}

Vaccination is an essential component for ensuring the health of those living with HIV. Since HIV-infected persons may have reduced initial and long-term responses post-vaccination, and hence may require alternate vaccination dosing 
strategies, specific guidelines for HIV-infected persons have been published to address these concerns. The current paper provides a concise summary of global and national vaccine recommendations for live, travel-related, and occupational-related vaccinations and highlights the role of these vaccines to protect HIV-infected adults from vaccine-preventable infectious diseases.

\section{ACKNOWLEDGEMENTS}

No funding or sponsorship was received for this study or publication of this article. All named authors meet the International Committee of Medical Journal Editors (ICMJE) criteria for authorship for this manuscript, take responsibility for the integrity of the work as a whole, and have given final approval for the version to be published.

Disclosures. Nancy F. Crum-Cianflone and Eva Sullivan have nothing to disclose, and both contributed to the writing this manuscript.

Compliance with ethics guidelines. This article is based on previously conducted studies and does not involve any new studies of human or animal subjects performed by either of the authors.

Open Access. This article is distributed under the terms of the Creative Commons Attribution-NonCommercial 4.0 International License (http://creativecommons.org/licenses/ by-nc/4.0/), which permits any noncommercial use, distribution, and reproduction in any medium, provided you give appropriate credit to the original author(s) and the source, provide a link to the Creative Commons license, and indicate if changes were made.

\section{REFERENCES}

1. World Health Organization Europe. In: Eramova I, Matic S, Munz M (eds) HIV/AIDS treatment and care: clinical protocols for the WHO European region. 2007. http://www.euro.who.int/_data/
assets/pdf_file/0004/78106/E90840.pdf. Accessed 30 Mar 2017.

2. World Health Organization. Vaccine position papers. http://www.who.int/immunization/ documents/positionpapers/en/. Accessed 30 Mar 2017.

3. British HIV association guidelines on the use of vaccines in HIV-positive adults 2015. http://www. bhiva.org/documents/Guidelines/Immunisation/ consultation/BHIVA-Immunisation-Guidelines2015-Consultation.pdf. Accessed 30 Mar 2017.

4. Kim DK, Riley LE, Harriman KH, Hunter P, Bridges $\mathrm{CB}$, On behalf of the Advisory Committee on Immunization Practices. Recommended immunization schedule for adults aged 19 years or older, United States, 2017. Ann Intern Med. 2017;166:209-19.

5. Panel on Opportunistic Infections in HIV-Infected Adults and Adolescents. Guidelines for the prevention and treatment of opportunistic infections in HIV-infected adults and adolescents: recommendations from the Centers for Disease Control and Prevention, the National Institutes of Health, and the HIV Medicine Association of the Infectious Diseases Society of America. https://aidsinfo.nih. gov/contentfiles/lvguidelines/adult_OI.pdf. Accessed 30 Mar 2017.

6. Rubin LG, Levin MJ, Ljungman P, et al. 2013 IDSA clinical practice guideline for vaccination of the immunocompromised host. Clin Infect Dis. 2014;58:309-18.

7. Aberg JA, Gallant JE, Ghanem KG, Emmanuel P, Zingman BS, Horberg MA. Primary care guidelines for the management of persons infected with HIV: 2013 update by the HIV medicine association of the Infectious Diseases Society of America. Clin Infect Dis. 2014;58:e1-34.

8. European AIDS Clinical Society (EACS) guidelines for the treatment of HIV. Version 8.2. January 2017. http://www.eacsociety.org/guidelines/eacs-guidelines/ eacs-guidelines.html. Accessed 30 Mar 2017.

9. Frésard A, Gagneux-Brunon A, Lucht F, Botelho-Nevers E, Launay O. Immunization of HIV-infected adult patients-French recommendations. Hum Vaccin Immunother. 2016;12:2729-41.

10. Kramer JM, LaRussa P, Tsai WC, et al. Disseminated vaccine strain varicella as the acquired immunodeficiency syndrome-defining illness in a previously undiagnosed child. Pediatrics. 2001;108:E39.

11. Maves RC, Tripp MS, Dell TG, et al. Disseminated vaccine-strain varicella as initial presentation of the acquired immunodeficiency syndrome: a case 
report and review of the literature. J Clin Virol. 2014;59:63-6.

12. Veenstra J, van Praag RM, Krol A, et al. Complications of varicella zoster virus reactivation in HIV-infected homosexual men. AIDS. 1996;10:393-9.

13. Moanna A, Rimland D. Decreasing incidence of herpes zoster in the highly active antiretroviral therapy era. Clin Infect Dis. 2013;57:122-5.

14. Blank LJ, Polydefkis MJ, Moore RD, Gebo KA. Herpes zoster among persons living with HIV in the current antiretroviral therapy era. J Acquir Immune Defic Syndr. 2012;61:203-7.

15. Grabar S, Tattevin P, Selinger-Leneman $H$, et al. Incidence of herpes zoster in HIV-infected adults in the combined antiretroviral therapy era: results from the FHDH-ANRS CO4 cohort. Clin Infect Dis. 2015;60:1269-77.

16. Wallace MR, Hooper DG, Pyne JM, Graves SJ, Malone JL. Varicella immunity and clinical disease in HIV-infected adults. South Med J. 1994;87:74-6.

17. Molton J, Smith C, Chaytor S, et al. Seroprevalence of common vaccine-preventable viral infections in HIV-positive adults. J Infect. 2010;61:73-80.

18. Aziz M, Kessler H, Huhn G. Providers' lack of knowledge about herpes zoster in HIV-infected patients is among barriers to herpes zoster vaccination. Int J STD AIDS. 2013;24:433-9.

19. Souty C, Boos E, Turbelin C, et al. Vaccination against varicella as post-exposure prophylaxis in adults: a quantitative assessment. Vaccine. 2015;33:446-50.

20. Son M, Shapiro ED, LaRussa P, et al. Effectiveness of varicella vaccine in children infected with HIV. J Infect Disl. 2010;201:1806-10.

21. Levin MJ, Gershon AA, Weinberg A, et al. Immunization of $\mathrm{HIV}$-infected children with varicella vaccine. J Pediatr. 2001;139:305-10.

22. Levin MJ, Gershon AA, Weinberg A, Song LY, Fentin $\mathrm{T}$, Nowak B. Administration of live varicella vaccine to HIV-infected children with current or past significant depression of CD4(+) T cells. J Infect Dis. 2006;194:247-55.

23. Taweesith W, Puthanakit T, Kowitdamrong E, et al. The immunogenicity and safety of live attenuated varicella-zoster virus vaccine in human immunodeficiency virus-infected children. Pediatr Infect Dis J. 2011;30:320-4.
24. Weinberg A, Levin MJ, Macgregor RR. Safety and immunogenicity of a live attenuated varicella vaccine in VZV-seropositive HIV-infected adults. Hum Vaccin. 2010;6:318-21.

25. Morrison VA, Johnson GR, Schmader KE, Shingles Prevention Study Group, et al. Long-term persistence of zoster vaccine efficacy. Clin Infect Dis. 2015;60:900-9.

26. Barnabas RV, Baeten JM, Lingappa JR, Partners in Prevention HSV/HIV Transmission Study Team, et al. Acyclovir prophylaxis reduces the incidence of herpes zoster among HIV-infected individuals: results of a randomized clinical trial. J Infect Dis. 2016;213:551-5.

27. Lal H, Cunningham AL, Godeaux O, ZOE-50 Study Group, et al. Efficacy of an adjuvanted herpes zoster subunit vaccine in older adults. $\mathrm{N}$ Engl J Med. 2015;372:2087-96.

28. Cunningham AL, Lal H, Kovac M, et al. Efficacy of the herpes zoster subunit vaccine in adults 70 years of age or older. N Engl J Med. 2016;375:1019-31.

29. Berkowitz EM, Moyle G, Stellbrink HJ, Zoster-015 $\mathrm{HZ} / \mathrm{su}$ Study Group, et al. Safety and immunogenicity of an adjuvanted herpes zoster subunit candidate vaccine in HIV-infected adults: a phase $1 / 2$ a randomized, placebo-controlled study. J Infect Dis. $2015 ; 211: 1279-87$.

30. Mullane KM, Winston DJ, Wertheim MS, et al. Safety and immunogenicity of heat-treated zoster vaccine (ZVHT) in immunocompromised adults. J Infect Dis. 2013;208:1375-85.

31. Oxman MN, Levin MJ, Johnson GR, Shingles Prevention Study Group, et al. A vaccine to prevent herpes zoster and postherpetic neuralgia in older adults. N Engl J Med. 2005;352:2271-84.

32. Benson CA, Hua L, Anderson JW, et al for the ACTG A5247 Team. Zostavax is generally safe and immunogenic in HIV + adults virologically suppressed on ART: results of a phase 2, randomized, double-blind, placebo-controlled trial. 19th CROI, Seattle, 5-8 Mar 2012. Oral abstract 96.

33. Shafran SD. Live attenuated herpes zoster vaccine for HIV-infected adults. HIV Med. 2016;17:305-10.

34. Dayan GH, Quinlisk MP, Parker AA, et al. Recent resurgence of mumps in the United States. N Engl J Med. 2008;358:1580-9.

35. Phadke VK, Bednarczyk RA, Salmon DA, Omer SB. Association between vaccine refusal and 
vaccine-preventable diseases in the United States: a review of measles and pertussis. JAMA. 2016;315:1149-58.

36. Kaplan LJ, Daum RS, Smaron M, McCarthy CA. Severe measles in immunocompromised patients. JAMA. 1992;267:1237-41.

37. Angel JB, Walpita P, Lerch RA, et al. Vaccine-associated measles pneumonitis in an adult with AIDS. Ann Intern Med. 1998;129:104-6.

38. Centers for Disease Control and Prevention (CDC). Measles pneumonitis following measles-mumps-rubella vaccination of a patient with HIV infection, 1993. Morb Mortal Wkly Rep. 1996;45:603-6.

39. Grabmeier-Pfistershammer K, Poeppl W, Herkner H, et al. High need for MMR vaccination in HIV infected adults in Austria. Vaccine. 2014;32:6020-3.

40. Llenas-García J, Rubio R, Hernando A, Arrazola P, Pulido F. Do HIV-positive adult immigrants need to be screened for measles-mumps-rubella and varicella zoster virus immunization? AIDS Care. 2013;25:980-9.

41. Palumbo P, Hoyt L, Demasio K, Oleske J, Connor E. Population-based study of measles and measles immunization in human immunodeficiency virus-infected children. Pediatr Infect Dis J. 1992;11:1008-14.

42. Wallace MR, Hooper DG, Graves SJ, Malone JL. Measles seroprevalence and vaccine response in HIV-infected adults. Vaccine. 1994;12:1222-4.

43. Kemper CA, Zolopa AR, Hamilton JR, Fenstersheib M, Bhatia G, Deresinski SC. Prevalence of measles antibodies in adults with HIV infection: possible risk factors of measles seronegativity. AIDS. 1992;6:1321-5.

44. Singh HK, Chiu YL, Wilkin T. Measles, mumps, and rubella serostatus and response to MMR vaccination among HIV-infected adults. AIDS Patient Care STDS. $2015 ; 29: 461-4$.

45. Sprauer MA, Markowitz LE, Nicholson JK, et al. Response of human immunodeficiency virus-infected adults to measles-rubella vaccination. J Acquir Immune Defic Syndr. 1993;6:1013-6.

46. Belaunzarán-Zamudio PF, García-León ML, Wong-Chew RM, et al. Early loss of measles antibodies after MMR vaccine among HIV-infected adults receiving HAART. Vaccine. 2009;27:7059-64.

47. Aurpibul L, Puthanakit T, Sirisanthana T, Sirisanthana V. Response to measles, mumps, and rubella revaccination in HIV-infected children with immune recovery after highly active antiretroviral therapy. Clin Infect Dis. 2007;45:637-42.

48. Abzug MJ, Qin M, Levin MJ, International Maternal Pediatric Adolescent AIDS Clinical Trials Group P1024 and P1061s Protocol Teams, et al. Immunogenicity, immunologic memory, and safety following measles revaccination in HIV-infected children receiving highly active antiretroviral therapy. J Infect Dis. 2012;206:512-22.

49. Aurpibul L, Puthanakit T, Sirisanthana T, Sirisanthana V. Persistence of measles, mumps, and rubella protective antibodies 3 years after revaccination in HIV-infected children receiving antiretroviral therapy. Clin Infect Dis. 2010;50:1415-8.

50. Stermole BM, Grandits GA, Roediger MP, Clark BM, Ganesan A, Weintrob AC, Crum-Cianflone NF, Ferguson TM, Macalino GE, Landrum ML. Long-term safety and serologic response to measles, mumps, and rubella vaccination in HIV-1 infected adults. Vaccine. 2011;29:2874-80.

51. Franco-Paredes C, Hidron A, Tellez I, Lesesne J, Del Rio C. HIV infection and travel: pretravel recommendations and health-related risks. Top HIV Med. 2009;17:2-11.

52. WHO: International Health and Travel. http:// www.who.int/ith/en/. Accessed 1 Apr 2017.

53. CDC Health Information for International Travel 2016 . http://wwwnc.cdc.gov/travel/page/ yellowbook-home-2014. Accessed 1 Apr 2017.

54. Khetsuriani N, Helfand R, Pallansch M, et al. Limited duration of vaccine poliovirus and other enterovirus excretion among human immunodeficiency virus infected children in Kenya. BMC Infect Dis. 2009;9:1-8.

55. Gouandjika-Vasilache I, Akoua-Koffi C, Begaud E, Dosseh A. No evidence of prolonged enterovirus excretion in HIV-seropositive patients. Trop Med Int Health. 2005;10:743-7.

56. Ion-Nedelcu N, Dobrescu A, Strebel PM, Sutter RW. Vaccine-associated paralytic poliomyelitis and HIV infection. Lancet. 1994;343:51-2.

57. Clinical update interim CDC guidance for travel to and from countries affected by the new polio vaccine requirements. https://wwwnc.cdc.gov/travel/ news-announcements/polio-guidance-new-requirem ents. Accessed 1 Apr 2017.

58. Kroon FP, van Dissel JT, Labadie J, van Loon AM, van Furth R. Antibody response to diphtheria, tetanus, and poliomyelitis vaccines in relation to the number of $\mathrm{CD} 4+\mathrm{T}$ lymphocytes in adults 
infected with human immunodeficiency virus. Clin Infect Dis. 1995;21:1197-203.

59. Vardinon N, Handsher R, Burke M, Zacut V, Yust I. Poliovirus vaccination responses in HIV-infected patients: correlation with T4 cell counts. J Infect Dis. 1990;162:238-41.

60. Barbi M, Biffi MR, Binda S, et al. Immunization in children with HIV seropositivity at birth: antibody response to polio vaccine and tetanus toxoid. AIDS. 1992;6:1465-9.

61. Gnanashanmugam D, Troy SB, Musingwini G, et al. Immunologic response to oral polio vaccine in human immunodeficiency virus-infected and uninfected Zimbabwean children. Pediatr Infect Dis J. 2012;31:176-80.

62. Troy SB, Kouiavaskaia D, Siik J, et al. Comparison of the immunogenicity of various booster doses of inactivated polio vaccine delivered intradermally versus intramuscularly to HIV-infected adults. J Infect Dis. 2015;211:1969-76.

63. Mathisen GE, Allen AD. Inactivated polio vaccine hyperimmunization in adults with HIV disease: a placebo-controlled study. AIDS. 1992;6:737-8.

64. Gordon MA, Kankwatira AM, Mwafulirwa G, et al. Invasive non-typhoid salmonellae establish systemic intracellular infection in HIV-infected adults: an emerging disease pathogenesis. Clin Infect Dis. 2010;50:953-62.

65. Gordon MA. Invasive nontyphoidal Salmonella disease: epidemiology, pathogenesis and diagnosis. Curr Opin Infect Dis. 2011;24:484-9.

66. Feasey NA, Houston A, Mukaka M, et al. A reduction in adult blood stream infection and case fatality at a large African hospital following antiretroviral therapy roll-out. PLoS ONE. 2014;9:e92226.

67. Jackson BR, Iqbal S, Mahon M. Updated recommendations for the use of typhoid vaccine-Advisory Committee on Immunization Practices, United States, 2015. Morb Mortal Wkly Rep. 2015;64:305-8.

68. Kroon FP, van Dissel JT, Ravensbergen E, Nibbering $\mathrm{PH}$, van Furth R. Impaired antibody response after immunization of HIV-infected individuals with the polysaccharide vaccine against Salmonella typhi (Typhim-Vi). Vaccine. 1999;17:2941-5.

69. Banda R, Yambayama V, Lalusha BD, et al. Safety of live, attenuated oral vaccines in HIV-infected Zambian adults-oral vaccines in HIV. Vaccine. 2012;30:5656-60.
70. Centers for Disease Control and Prevention. Yellow fever. https://wwwnc.cdc.gov/travel/yellowbook/ 2016/infectious-diseases-related-to-travel/yellowfever. Accessed 1 May 2017.

71. Smith DS. Travel medicine and vaccines for HIV-infected travelers. HIV Travel. 2012;20:111-5.

72. Barte H, Horvath TH, Rutherford GW. Yellow fever vaccine for patients with HIV infection. Cochrane Database Syst Rev. 2014;doi:10.1002/ 14651858.CD010929.pub2.

73. Sidibe M, Yactayo S, Kalle A, et al. Immunogenicity and safety of yellow fever vaccine among 115 HIV-infected patients after a preventive immunisation campaign in Mali. Trans R Soc Trop Med Hyg. 2012;106:437-44.

74. Sibailly TS, Wiktor SZ, Tsai TF, et al. Poor antibody response to yellow fever vaccination in children infected with human immunodeficiency virus type 1. Pediatr Infect Dis J. 1997;16:1177-9.

75. Pistone T, Verdière $\mathrm{CH}$, Receveur MC, Ezzedine K, Lafon ME, Malvy D. Immunogenicity and tolerability of yellow fever vaccination in 23 French HIV-infected patients. Curr HIV Res. 2010;8:461-6.

76. Tattevin P, Depatureaux AG, Chapplain JM, et al. Yellow fever vaccine is safe and effective in HIV-infected patients. AIDS. 2004;18:825-7.

77. Pacanowski J, Lacombe K, Campa P, et al. Plasma HIV-RNA is the key determinant of long-term antibody persistence after Yellow fever immunization in a cohort of 364 HIV-infected patients. J Acquir Immune Defic Syndr. 2012;59:360-7.

78. Veit O, Niedrig M, Chapuis-Taillard C, et al. Immunogenicity and safety of yellow fever vaccination for $102 \mathrm{HIV}$-infected patients. Clin Infect Dis. 2009;48:659-66.

79. Kengsakul K, Sathirapongsasuti K, Punyagupta S. Fatal myeloencephalitis following yellow fever vaccination in a case with HIV infection. J Med Assoc Thai. 2002;85:131-4.

80. Chapter 8: advising travelers with specific needs. Immunocompromised travelers. https://wwwnc. cdc.gov/travel/yellowbook/2016/advising-travelerswith-specific-needs/immunocompromised-travelers. Accessed 1 Apr 2017.

81. Pancharoen C, Thisyakorn U, Tantawichien T, Jaijaroensup W, Khawplod P, Wilde H. Failure of preand postexposure rabies vaccinations in a child infected with HIV. Scand J Infect Dis. 2001;33:390-1. 
82. Thisyakorn U, Pancharoen C, Wilde H. Immunologic and virologic evaluation of HIV-1-infected children after rabies vaccination. Vaccine. 2001;19:1534-7.

83. Thisyakorn U, Pancharoen C, Ruxrungtham K, et al. Safety and immunogenicity of preexposure rabies vaccination in children infected with human immunodeficiency virus type 1. Clin Infect Dis. 2000;30:218.

84. Tantawichien T, Jaijaroensup W, Khawplod P, Sitprija V. Failure of multiple-site intradermal postexposure rabies vaccination in patients with human immunodeficiency virus with low CD4+ T lymphocyte counts. Clin Infect Dis. 2001;33:E122-4.

85. Jaijaroensup W, Tantawichien T, Khawplod P, Tepsumethanon $\mathrm{S}$, Wilde $\mathrm{H}$. Postexposure rabies vaccination in patients infected with human immunodeficiency virus. Clin Infect Dis. 1999;28:913-4.

86. Gelinck LB, Jol-van der Zijde CM, Jansen-Hoogendijk AM, et al. Restoration of the antibody response upon rabies vaccination in HIV-infected patients treated with HAART. AIDS. 2009;23:2451-8.

87. Azzoni L, Foulkes AS, Firnhaber C, Yin X, Xiang ZQ, Li Y, Stevens W, Gross R, Ertl HC, Sanne I, Montaner LJ. Antiretroviral therapy interruptions result in loss of protective humoral immunity to neoantigens in HIV-infected individuals. AIDS. 2012;26:1355-62.

88. Sirikwin S, Likanonsakul S, Waradejwinyoo S, et al. Antibody response to an eight-site intradermal rabies vaccination in patients infected with human immunodeficiency virus. Vaccine. 2009;27:4350-4.

89. Rojanasuphot S, Shaffer N, Chotpitayasunondh T, et al. Response to JE vaccine among HIV-infected children, Bangkok, Thailand. Southeast Asian J Trop Med Public Health. 1998;29:443-50.

90. Puthanakit T, Aurpibul L, Yoksan S, Sirisanthana T, Sirisanthana V. Japanese encephalitis vaccination in HIV-infected children with immune recovery after highly active antiretroviral therapy. Vaccine. 2007;25:8257-61.

91. Puthanakit T, Aurpibul L, Yoksan S, Sirisanthana T, Sirisanthana V. A 3-year follow-up of antibody response in HIV-infected children with immune recovery vaccinated with inactivated Japanese encephalitis vaccine. Vaccine. 2010;28:5900-2.

92. Chokephaibulkit K, Plipat N, Yoksan S, et al. A comparative study of the serological response to Japanese encephalitis vaccine in HIV-infected and uninfected Thai children. Vaccine. 2010;28:3563-6.
93. von Seidlein L, Wang XY, Macuamule A, et al. Is HIV infection associated with an increased risk for cholera? Findings from a case-control study in Mozambique. Trop Med Int Health. 2008;13:683-8.

94. Lucas ME, Deen JL, von Seidlein L, et al. Effectiveness of mass oral cholera vaccination in Beira, Mozambique. N Engl J Med. 2005;352:757-67.

95. Lewis DJ, Gilks CF, Ojoo S, et al. Immune response following oral administration of cholera toxin B subunit to HIV-1-infected UK and Kenyan subjects. AIDS. 1994;8:779-85.

96. Ivers LC, Charles RC, Hilaire IJ, et al. Immunogenicity of the bivalent oral cholera vaccine Shanchol in Haitian adults with HIV infection. J Infect Dis. 2015;212:779-83.

97. Perry RT, Plowe CV, Koumare B, et al. A single dose of live oral cholera vaccine CVD 103-HgR is safe and immunogenic in HIV-infected and HIV-noninfected adults in Mali. Bull World Health Organ. 1998;76:63-71.

98. Westrop SJ, Moyle G, Jackson A, Nelson M, Mandalia S, Imami N. CCR5 antagonism impacts vaccination response and immune profile in HIV-1 infection. Mol Med. 2012;18:1240-8.

99. Amicizia D, Domnich A, Panatto D, et al. Epidemiology of tick-borne encephalitis (TBE) in Europe and its prevention by available vaccines. Hum Vaccin Immunother. 2013;9:1163-71.

100. Kindberg E, Mickiene A, Ax C, et al. A deletion in the chemokine receptor 5 (CCR5) gene is associated with tickborne encephalitis. J Infect Dis. 2008;197:266-9.

101. Panasiuk B, Prokopowicz D, Panasiuk A. Immunological response in HIV-positive patients vaccinated against tick-borne encephalitis. Infection. 2003;31:45-6.

102. Wolf HM, Pum M, Jáger R, István L, Mannhalter JW, Eibl MM. Cellular and humoral immune responses in haemophiliacs after vaccination against tick-borne encephalitis. Br J Haematol. 1992;82:374-83.

103. Wright JG, Quinn CP, Shadomy S, Messonnier N, Centers for Disease Control and Prevention (CDC). Use of anthrax vaccine in the United States: recommendations of the Advisory Committee on Immunization Practices (ACIP), 2009. Recomm Rep. 2010;59:1-30.

104. Bartlett JG. Smallpox vaccination and patients with human immunodeficiency virus infection or acquired immunodeficiency syndrome. Clin Infect Dis. 2003;36:468-71. 
105. Petersen BW, Damon IK, Pertowski CA, et al. Clinical guidance for smallpox vaccine use in a postevent vaccination program. Recomm Rep. $2015 ; 64: 1-26$.

106. Tasker SA, Schnepf GA, Lim M, US Department of Defense Tri-Service AIDS Clinical Consortium, et al. Unintended smallpox vaccination of HIV-1-infected individuals in the United States military. Clin Infect Dis. 2004;38:1320-2.

107. Redfield RR, Wright DC, James WD, Jones TS, Brown C, Burke DS. Disseminated vaccinia in a military recruit with human immunodeficiency virus (HIV) disease. N Engl J Med. 1987;316:673-6.

108. Greenberg RN, Overton ET, Haas DW, et al. Safety, immunogenicity, and surrogate markers of clinical efficacy for modified vaccinia Ankara as a smallpox vaccine in $\mathrm{HIV}$-infected subjects. J Infect Dis. 2013;207:749-58.

109. Cosma A, Nagaraj R, Staib C, et al. Evaluation of modified vaccinia virus Ankara as an alternative vaccine against smallpox in chronically HIV type 1-infected individuals undergoing HAART. AIDS Res Hum Retrovir. 2007;23:782-93.

110. Overton ET, Stapleton J, Frank I, et al. Safety and immunogenicity of modified vaccinia Ankara-Bavarian Nordic smallpox vaccine in vaccinia-naïve and experienced human immunodeficiency virus-infected individuals: an open-label, controlled clinical phase II trial. Open Forum Infect Dis. 2015;2:ofv040.

111. Collins S, Ramsay M, Campbell H, Slack MP, Ladhani SN. Invasive Haemophilus influenzae type $b$ disease in England and Wales: who is at risk after 2 decades of routine childhood vaccination? Clin Infect Dis. 2013;57:1715-21.

112. Steinhart R, Reingold AL, Taylor F, Anderson G, Wenger JD. Invasive Haemophilus influenzae infections in men with HIV infection. JAMA. 1992;268:3350-2.

113. Steinhoff MC, Auerbach BS, Nelson KE, et al. Antibody responses to Haemophilus influenzae type $\mathrm{B}$ vaccines in men with human immunodeficiency virus infection. N Engl J Med. 1991;325:1837-42.

114. Dockrell DH, Poland GA, Steckelberg JM, Wollan PC, Strickland SR, Pomeroy C. Immunogenicity of three Haemophilus influenzae type b protein conjugate vaccines in HIV seropositive adults and analysis of predictors of vaccine response. Vaccine. 1999;17:2779-85.
115. De Sousa dos Santos S, Lopes MH, Simonsen V, Caiaffa Filho HH. Haemophilus influenzae type $b$ immunization in adults infected with the human immunodeficiency virus. AIDS Res Hum Retrovir. 2004;20:493-6.

116. Bruchfeld J, Correia-Neves M, Källenius G. Tuberculosis and HIV coinfection. Cold Spring Harb Perspect Med. 2015;5:a017871.

117. Pawlowski A, Jansson M, Sköld M, Rottenberg ME, Källenius G. Tuberculosis and HIV co-infection. PLoS Pathog. 2012;8:e1002464.

118. Mangtani P, Abubakar I, Ariti C, et al. Protection by BCG vaccine against tuberculosis: a systematic review of randomized controlled trials. Clin Infect Dis. $2014 ; 58: 470-80$.

119. Centers for Disease Control (CDC). Disseminated Mycobacterium bovis infection from BCG vaccination of a patient with acquired immunodeficiency syndrome. Morb Mortal Wkly Rep. 1985;34:227-8.

120. Boudes P, Sobel A, Deforges L, Leblic E. Disseminated Mycobacterium bovis infection from BCG vaccination and HIV infection. JAMA. 1989;262:2386.

121. Vera JH, Hill SC, Rubinstein L. Bacille Calmette-Guerin disease following Bacille Calmette-Guerin vaccination of an HIV-infected health-care worker. Int J STD AIDS. 2012;23:e1-2.

122. Azzopardi P, Bennett CM, Graham SM, Duke T. Bacille Calmette-Guérin vaccine-related disease in HIV-infected children: a systematic review. Int J Tuberc Lung Dis. 2009;13:1331-44.

123. Reynes J, Perez C, Lamaury I, Janbon F, Bertrand A. Bacille Calmette-Guérin adenitis 30 years after immunization in a patient with AIDS. J Infect Dis. 1989;160:727.

124. Armbruster C, Junker W, Vetter N, Jaksch G. Disseminated bacille Calmette-Guérin infection in an AIDS patient 30 years after BCG vaccination. J Infect Dis. 1990;162:1216.

125. Lumb R, Shaw D. Mycobacterium bovis (BCG) vaccination. Progressive disease in a patient asymptomatically infected with the human immunodeficiency virus. Med J Aust. $1992 ; 156: 286-7$.

126. Smith E, Thybo S, Bennedsen J. Infection with $M y$ cobacterium bovis in a patient with AIDS: a late complication of BCG vaccination. Scand J Infect Dis. 1992;24:109-10. 